\title{
Repurposed agents in the Alzheimer's disease drug development pipeline
}

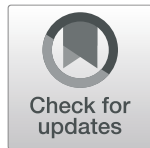

Justin Bauzon', Garam Lee ${ }^{2}$ and Jeffrey Cummings ${ }^{2,3^{*}}$ (D)

\begin{abstract}
Background: Treatments are needed to address the growing prevalence of Alzheimer's disease (AD). Clinical trials have failed to produce any AD drugs for Food and Drug Administration (FDA) approval since 2003, and the pharmaceutical development process is both time-consuming and costly. Drug repurposing provides an opportunity to accelerate this process by investigating the AD-related effects of agents approved for other indications. These drugs have known safety profiles, pharmacokinetic characterization, formulations, doses, and manufacturing processes.
\end{abstract}

Methods: We assessed repurposed AD therapies represented in Phase I, Phase II, and Phase III of the current AD pipeline as registered on ClinicalTrials.gov as of February 27, 2020.

Results: We identified 53 clinical trials involving 58 FDA-approved agents. Seventy-eight percent of the agents in trials had putative disease-modifying mechanisms of action. Of the repurposed drugs in the pipeline $20 \%$ are hematologic-oncologic agents, $18 \%$ are drugs derived from cardiovascular indications, $14 \%$ are agents with psychiatric uses, $12 \%$ are drug used to treat diabetes, $10 \%$ are neurologic agents, and the remaining $26 \%$ of drugs fall under other conditions. Intellectual property strategies utilized in these programs included using the same drug but altering doses, routes of administration, or formulations. Most repurposing trials were supported by Academic Medical Centers and were not funded through the biopharmaceutical industry. We compared our results to a European trial registry and found results similar to those derived from ClinicalTrials.gov.

Conclusions: Drug repurposing is a common approach to AD drug development and represents 39\% of trials in the current AD pipeline. Therapies from many disease areas provide agents potentially useful in AD. Most of the repurposed agents are generic and a variety of intellectual property strategies have been adopted to enhance their economic value.

Keywords: Alzheimer's disease, Clinical trials, Repurposing, Dose, Formulation, Disease-modification

\section{Background}

Alzheimer's disease $(\mathrm{AD})$ is a progressive neurodegenerative disorder with a rising prevalence due to the global increase in the aging population [1]. It is estimated that one in 85 people will be living with an AD diagnosis by

\footnotetext{
* Correspondence: jcummings@cnsinnovations.com

${ }^{2}$ Cleveland Clinic Lou Ruvo Center for Brain Health, Las Vegas, NV 89106, USA ${ }^{3}$ Chambers-Grundy Center for Transformative Neuroscience, Department of Brain Health, School of Integrated Health Sciences, University of Nevada, Las Vegas (UNLV), Las Vegas, NV 89154, USA

Full list of author information is available at the end of the article
}

2050 [2]. An urgent need exists to identify effective interventions that will combat this disabling and ultimately fatal disease. Attempts to find new therapies for $\mathrm{AD}$ have been made by academic centers and biopharmaceutical companies: identifying new chemical entities, optimizing them for human use, and investigating their effects in clinical trials. However, the lack of an approved drug for AD by the Food and Drug Administration (FDA) since 2003 demonstrates the complexity of this objective [3]. 
Efforts to produce new treatments for AD are focused primarily on identifying new compounds that may improve symptoms, slow disease progression, delay the onset, or ultimately prevent the disease. An alternative approach to advancing new treatments is the development of drugs approved for a non-AD-related indication that may impact the biology of AD through on-target or off-target mechanisms. This repurposing strategy has succeeded in other conditions and has promise for $A D$ since many FDA-approved agents exhibit AD-relevant effects evident through in vitro, animal, epidemiologic, and observational studies [4-9].

To better understand repurposing in the AD drug development process, we conducted an analysis of all ongoing $\mathrm{AD}$ clinical trials involving FDA-approved drugs. We analyzed trial data reported on ClinicalTrials.gov, a comprehensive US government database with mandated registration of all clinical trials conducted within the US. We examined all currently ongoing trials that involved repurposed agents and compared this to all drugs in the AD pipeline [10-14]. We also compared ongoing trial activity of repurposed $\mathrm{AD}$ drugs as reported on the European Union Clinical Trials Register (EUCTR). Our goal was to examine how repurposing efforts in the $A D$ drug pipeline compares to overall drug development activity in the United States (US) and international pipelines and to provide insight into repurposing as a drug development strategy.

\section{Methods}

This study is based on the activity of clinical trials reported on ClinicalTrials.gov. By US law, all clinical trials conducted within the US must be registered within 21 days of the enrollment of the first participant $[15,16]$. ClinicalTrials.gov is a comprehensive and valid data source for the study of clinical trials conducted in the US. Most, but not all, non-US trials are registered on clinicaltrials.gov; Phase I trials conducted outside the US are often not registered and our findings may underrepresent the agents populating global Phase I efforts.

We used a previously constructed trial database that included the data of interest [10-14] and report on all trials currently testing repurposed agents in Phases I, II, and III. The results reported here are based on trials registered on ClinicalTrials.gov as of February 27, 2020. We used ClinicalTrials.gov labeling and included trials that were recruiting, active but not recruiting, enrolling by invitation, and not yet recruiting. We do not include trials of stem cell therapies among the interventions reviewed.

Our focus on repurposed agents places an importance on drugs with an FDA-approved indication(s). Agents were considered to be repurposed based on the information recorded on Drugs@FDA (fda.gov/drugsatfda), a US government database containing drug products approved since 1939 that is updated daily [17]. The approved use for each drug was determined by the most recent label provided by Drugs@FDA. Some drugs have more than one FDA-approved indication, and this is noted.

Agents were further classified based on the therapeutic area for which each drug is prescribed as determined by its approved indication. Based on this classification, we examined the therapeutic area origin of agents being studied across all AD trials. Some agents are being studied in multiple ongoing trials; we defined these agents as "unique" agents. Varieties of a particular compound seen in two or more trials (i.e., dosing, routes of administration) were noted.

Some trials are investigating multiple repurposed drugs in separate treatment arms (e.g., telmisartan vs perindopril) or in combination (e.g., losartan, amlodipine, and atorvastatin as a single treatment). For the purposes of this study, we treat the former as separate therapeutic trial agents; the latter is considered a single agent with respect to its clinical trial because the intent of the trial is to study the effects of the fixed combination as a single therapy.

A drug's putative mechanism of action (MOA) in $\mathrm{AD}$ was determined from the information on ClinicalTrials. gov or from a comprehensive review of the literature for the specific agent. We grouped the mechanisms into disease-modifying therapies (DMTs) or symptomatic agents. The latter is further divided into cognitiveenhancers or those that address neuropsychiatric and behavioral symptoms. We identified an agent as a DMT when the purpose of the intervention was to modify the underlying disease biology and to delay the onset or slow the progression of the disease. DMTs were divided into those targeting amyloid-related targets, tau-related mechanisms, and those with "other" mechanisms (i.e., neuroprotection, anti-inflammation, metabolic effects). The distinction between symptomatic agents and DMTs can be arbitrary and some agents may have both properties. For the purposes of this study, we chose what appears to be the principal MOA.

We retrospectively compare current repurposing trial data with historical data from previous $\mathrm{AD}$ pipeline reviews, starting from 2016 onward [10-14]. We used identical methodology to ascertain inclusion of clinical trials as detailed above.

Our study analyzes parallel activity between the US and European $\mathrm{AD}$ pipelines using trial information reported on the EUCTR. This public register enables access to data from the European Union Drug Regulating Authorities Clinical Trials (EudraCT) database, which contains protocols and results of studies conducted in the European Union (EU) or the European Economic Area (EEA). We used EUCTR to query Phase I, Phase II, 
and Phase III AD trials with ongoing status recorded as of February 27, 2020. We distinguish clinical trials exclusive to the EU/EEA from overlap studies running concurrently in the US.

\section{Results}

\section{Overview}

There are 58 total repurposed drugs in 53 clinical trials as reported on ClinicalTrials.gov. We identified 15 agents in 12 trials in Phase III, 33 agents in 32 trials in Phase II, and 10 agents in nine trials in Phase I. Of these trials, six agents were in Phase II/III trials and two agents were in Phase I/II trial. Several trials have more than one agent; some drugs are being studied across multiple trials.

Analysis of the FDA-approved indications of all repurposed drugs in the pipeline showed that $20 \%$ are hematologic-oncologic agents, $18 \%$ cardiovascular agents, $14 \%$ are psychiatric agents, $12 \%$ are antidiabetic treatments, $10 \%$ are drugs for other neurologic disorders, and the remaining $26 \%$ of drugs are derived from other therapeutic area categories. Repurposed psychiatric drugs in Phase III trials represent $50 \%$ of all agents being assessed for the treatment of neuropsychiatric symptoms in AD. Half of all cardiovascular agents under study are in Phase III trials. The majority of the pipeline's hematologic-oncologic, antidiabetic, and neurologic agents are in Phase II trials (70, 67, and 67\%, respectively). Phase II has more repurposed agents than Phase I and Phase III combined. Figure 1 shows the classification of all repurposed drugs by FDA-approved indications within their respective clinical phases.

Review of the AD-related MOAs of all agents shows that $78 \%$ are being developed as DMTs, $16 \%$ are symptomatic agents addressing neuropsychiatric and behavioral changes, and $7 \%$ are cognitive enhancers (Table 1). Escitalopram is being investigated separately as a cognitive enhancer in Phase I and as a neuropsychiatric agent in Phase III. Among DMT trials, the agents are derived primarily from hematologic-oncologic, cardiovascular, and antidiabetic indications $(26,18$, and $16 \%$, respectively). In contrast, symptomatic agents are derived mostly from treatments addressing psychiatric illness (50\%). Figure 2 shows the repurposed agents' AD-related MOAs.

\section{Unique repurposed agents}

There are 50 unique repurposed drugs (defined as drugs represented at least once from the 58 total in the pipeline) that are under study among all $\mathrm{AD}$ pipeline trials. Twenty percent are hematologic-oncologic agents, 18\% are cardiovascular agents, $14 \%$ are psychiatric agents, $12 \%$ are antidiabetic agents, $10 \%$ are neurologic drugs, and the remaining $26 \%$ of treatments were used in other categories. Figure 3 provides a graphical summary of the classifications of all unique repurposed agents represented in the current $\mathrm{AD}$ pipeline.

The agent most represented among all trials is levetiracetam (four Phase II trials, one Phase III trial). Telmisartan, montelukast, escitalopram, and valacyclovir are each in multiple Phase II and Phase III trials. A propriety

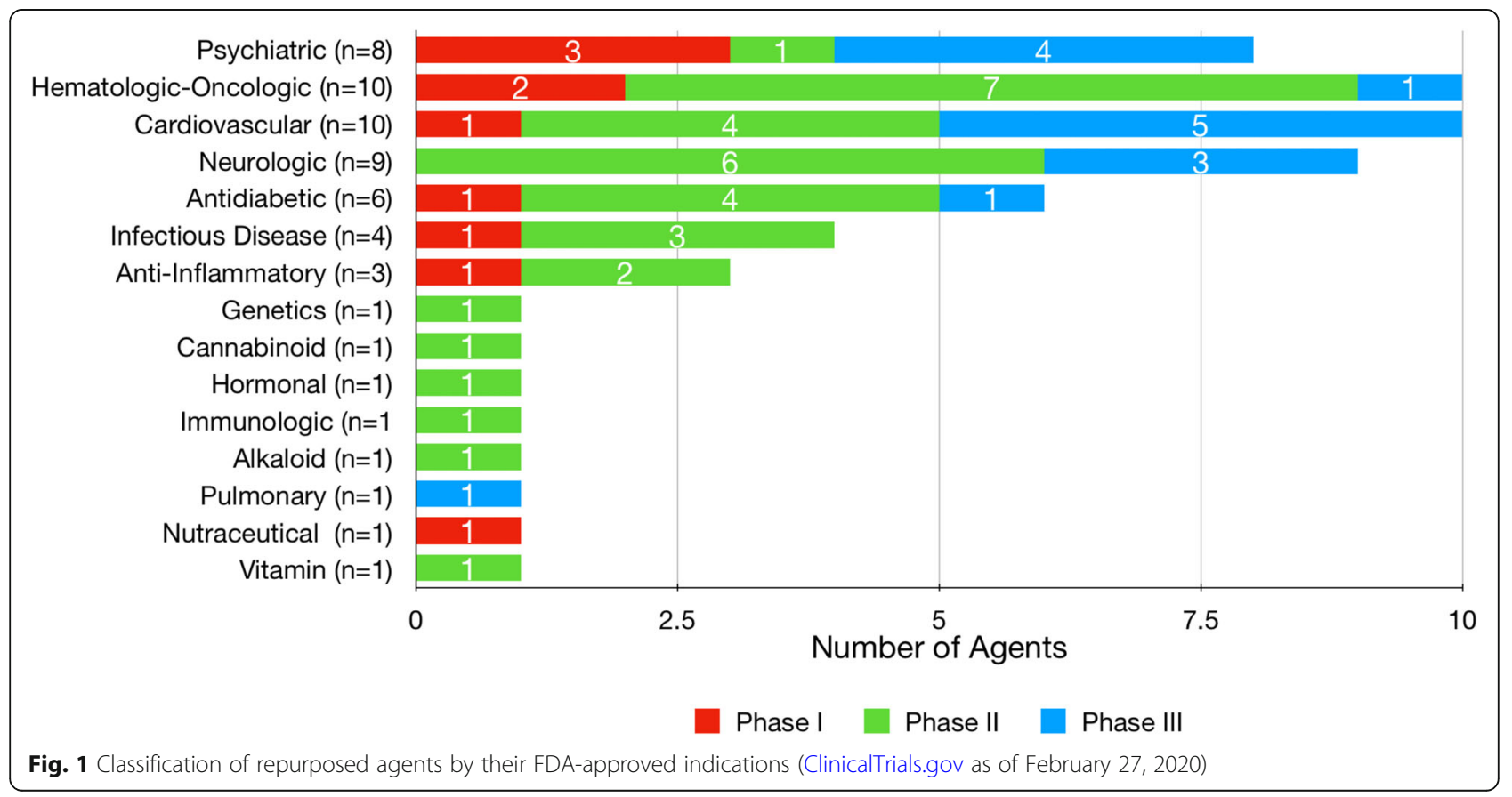


Table 1 Alzheimer's disease-related mechanisms of action of repurposed agents in the pipeline (ClinicalTrials.gov as of February 27, 2020)

\begin{tabular}{|c|c|c|c|c|c|}
\hline $\begin{array}{l}\text { Approved therapeutic } \\
\text { area }\end{array}$ & $\begin{array}{l}\text { Disease-modifying } \\
\text { therapies }\end{array}$ & $\begin{array}{l}\text { Cognitive } \\
\text { enhancers }\end{array}$ & $\begin{array}{l}\text { Behavioral } \\
\text { symptoms }\end{array}$ & $\begin{array}{l}\text { Behavioral + cognitive } \\
\text { symptoms }\end{array}$ & $\begin{array}{l}\text { Total \# of agents } \\
(\%)\end{array}$ \\
\hline Hematologic-oncologic & 10 & - & - & - & $10(20.0)$ \\
\hline Cardiovascular & 7 & 1 & 1 & - & $9(18.0)$ \\
\hline Psychiatric & 1 & 1 & 4 & 1 & $7(14.0)$ \\
\hline Antidiabetic & 6 & - & - & - & $6(12.0)$ \\
\hline Neurologic & 3 & - & 2 & - & $5(10.0)$ \\
\hline Infectious disease & 3 & - & - & - & $3(6.0)$ \\
\hline Anti-inflammatory & 2 & - & - & - & $2(4.0)$ \\
\hline Genetics & 1 & - & - & - & $1(2.0)$ \\
\hline Cannabinoid & - & - & 1 & - & $1(2.0)$ \\
\hline Hormonal & 1 & - & - & - & $1(2.0)$ \\
\hline Immunologic & 1 & - & - & - & $1(2.0)$ \\
\hline Alkaloid & - & 1 & - & - & $1(2.0)$ \\
\hline Pulmonary & 1 & - & - & - & $1(2.0)$ \\
\hline Nutraceutical & 1 & - & - & - & $1(2.0)$ \\
\hline Vitamin & 1 & - & - & - & $1(2.0)$ \\
\hline TOTAL & 38 & 3 & 8 & 1 & $50(100.0)$ \\
\hline
\end{tabular}

low-dose formulation of levetiracetam (AGB101, which is $1 / 15$ th of the original dose) is being investigated in Phase II and Phase III trials.

\section{Phase III}

In Phase III trials, there are 15 repurposed agents in 12 trials (Table 2); this represents one-third of all Phase III trials in the AD pipeline [14]. These include eight DMTs, one cognitive-enhancer, and six agents addressing behavioral symptoms. Among DMT agents, one had amyloid-related targets, five had anti-inflammatory MOAs, four were considered metabolic, and two were neuroprotective agents.

Therapeutic areas represented among the repurposed agents included five cardiovascular drugs, four with psychiatric indications, three neurological drugs, one hematologic-oncologic agent, one antidiabetic agent, and one pulmonary agent. Among these drugs, sedativehypnotics were the most common.
In Phase III, there were two prevention trials with cognitively normal (preclinical) participants; three trials of patients with prodromal $\mathrm{AD} /$ mild cognitive impairment (MCI); five trials of patients with mild to moderate AD; and two trials of patients with mild to moderate/severe $\mathrm{AD}$.

\section{Phase II}

Phase II of the AD pipeline has 32 trials involving 33 repurposed agents (Table 3); this is $44 \%$ of all $\mathrm{AD}$ trials in Phase II [14]. Of the repurposed agents, there were 27 DMTs, one cognitive-enhancing agent, and three drugs for behavioral symptoms. Among the DMTs, three involve amyloid targets, one addresses tau-related targets, one has a mechanism relevant to both amyloid- and taurelated targets, and 22 have other MOAs (e.g., neuroprotection, metabolic, or anti-inflammatory). Of the symptomatic agents, all four are neurotransmitter-based.

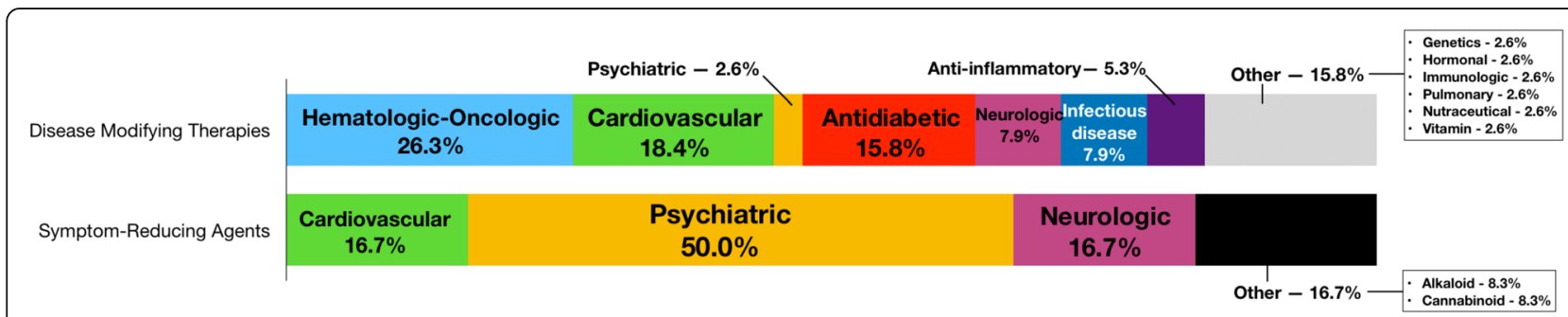

Fig. 2 Percentage of repurposed agents currently under study by mechanisms of action (ClinicalTrials.gov as of February 27, 2020) 


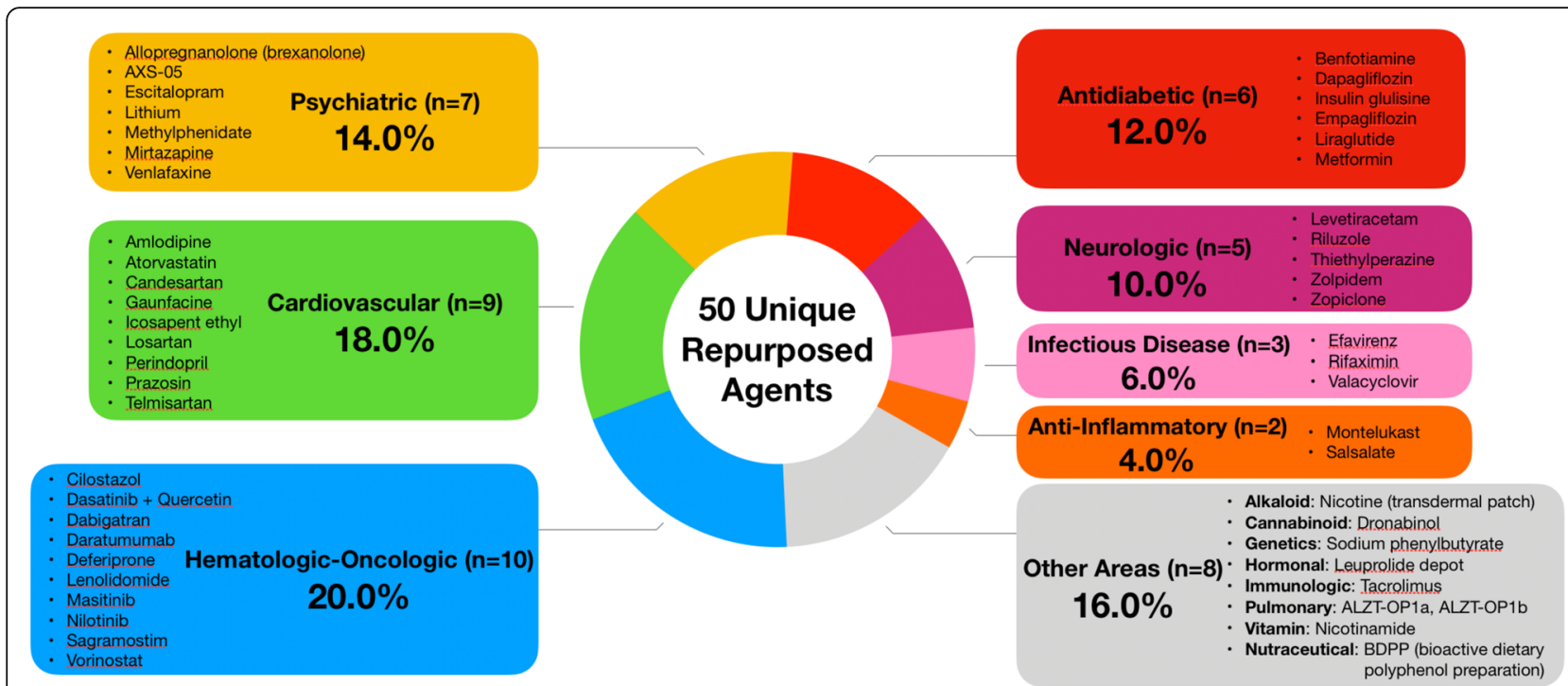

Fig. 3 Classification of the therapeutic areas of repurposed agent currently under study (ClinicalTrials.gov as of February 27, 2020)

Therapeutic areas of Phase II repurposed agents comprise hematologic-oncologic agents (seven), neurologic drugs (six), cardiovascular drugs (four), antidiabetic agents (four), and other classes (e.g., cannabinoid, alkaloid; 12 total). Anticonvulsants were the most represented drug class in Phase II.

There is one prevention trial; 19 trials of patients with prodromal/MCI or prodromal/mild-AD; 11 trials in mild-moderate $\mathrm{AD}$; and one trial of patients with mildmoderate/severe AD. Among DMT trials, there is one prevention trial with cognitively normal participants, 17 for prodromal/mild $\mathrm{AD}$, and eight for participants with mild-moderate AD.

\section{Phase I}

There are ten repurposed agents among nine Phase I trials (Table 4), which is $33 \%$ of Phase I trials in the pipeline [14]. Of these, there are eight DMTs and two cognitive enhancers. No agents addressing neuropsychiatric symptoms are represented in the Phase I pipeline. Of the DMTs in Phase I, one agent is directed at amyloid-related targets while seven have other MOAs (e.g., neuroprotection, metabolic, or anti-inflammatory). No tau-related repurposed agents are under investigation in Phase I of the current pipeline.

In Phase I, repurposed agents include three psychiatric drugs, two hematologic-oncologic agents, one cardiovascular drug, one antidiabetic agent, and three agents in other drug classes (e.g., anti-inflammatory drug, infectious disease agent).

\section{Trial sponsors}

Across all trials of repurposed agents, 9\% are sponsored by the biopharma industry, $79 \%$ by Academic Medical
Centers (with funding from the National Institutes of Health, industry, and/or other entities such as a consortium or a philanthropic foundation), and $11 \%$ by others. Table 5 shows sponsors for each phase of development.

\section{Repurposed agents in the pipeline from 2016 to 2020}

Table 6 summarizes the number of repurposing trials in previous annual AD pipeline reports [10-14]. Since 2016, the number of trials involving repurposed agents has increased by $89 \%$. The number of trials has grown each year, with the greatest rate of increase occurring between 2019 and 2020 (23\% growth). Between 2016 and 2020, repurposed DMT trials have increased 180\%; repurposed trials focused on symptom-reduction have decreased by $67 \%$.

Phase I trials of repurposed agents have increased 125\% since 2016; Phase II and Phase III trials have risen $88 \%$ and $71 \%$, respectively. DMT trials of repurposed agents currently make up $79 \%$ of all repurposed agents in the current pipeline (up from 54\% in 2016; Fig. 4).

\section{Repurposed agents in the European $A D$ pipeline}

Out of $138 \mathrm{AD}$ trials with an ongoing status registered with the EUCTR, we identified 14 trials involving 14 FDA-approved drugs (Table 7). Eleven of these were dual-registered with the US and EU/EEA databases. Five trials were in Phase III and nine were in Phase II. Six had a completed or terminated status when compared to the information listed on ClinicalTrials.gov. Three trials were registered exclusively with the EUCTR: epoetin alfa, levodopa-carbidopa, and cannabidiol.

There were six DMTs, five cognitive-enhancing agents, and three drugs addressing neuropsychiatric symptoms among the repurposed agents. Therapeutic 
Table 2 Repurposed agents currently in Phase III in the Alzheimer's disease development pipeline (as of February 27, 2020)

\begin{tabular}{|c|c|c|c|c|c|c|c|}
\hline Agent name & Phase & $\begin{array}{l}\text { Agent } \\
\text { mechanism class }\end{array}$ & $\begin{array}{l}\text { Therapeutic } \\
\text { purpose under } \\
\text { study }\end{array}$ & Drug class & $\begin{array}{l}\text { FDA-approved } \\
\text { indication(s) }\end{array}$ & $\begin{array}{l}\text { Therapeutic } \\
\text { field }\end{array}$ & $\begin{array}{l}\text { ClinicalTrials.gov } \\
\text { ID(s) (EudraCT } \\
\text { ID) }\end{array}$ \\
\hline $\begin{array}{l}\text { ALZT-OP1a, } \\
\text { ALZT-OP1b (cro- } \\
\text { molyn with and } \\
\text { without } \\
\text { ibuprofen) }\end{array}$ & III & $\begin{array}{l}\text { Anti-amyloid, } \\
\text { anti-inflammatory }\end{array}$ & $\begin{array}{l}\text { Reduce neuronal } \\
\text { damage; mast cells } \\
\text { may also play a role } \\
\text { in amyloid } \\
\text { pathology (DMT) }\end{array}$ & Mast cell stabilizer & $\begin{array}{l}\text { Bronchial asthma; } \\
\text { mastocytosis }\end{array}$ & Pulmonary & $\begin{array}{l}\text { NCT02547818 } \\
(2015-002147- \\
34)\end{array}$ \\
\hline Amlodipine & II/III & $\begin{array}{l}\text { Anti- } \\
\text { inflammatory, } \\
\text { metabolic }\end{array}$ & $\begin{array}{l}\text { Vascular risk } \\
\text { reduction to } \\
\text { preserve cognitive } \\
\text { function (DMT) }\end{array}$ & $\begin{array}{l}\text { Calcium channel } \\
\text { blocker }\end{array}$ & $\begin{array}{l}\text { Hypertension; coronary } \\
\text { artery disease }\end{array}$ & Cardiovascular & NCT02913664 \\
\hline Atorvastatin & II/III & $\begin{array}{l}\text { Anti- } \\
\text { inflammatory, } \\
\text { metabolic }\end{array}$ & $\begin{array}{l}\text { Vascular risk } \\
\text { reduction to } \\
\text { preserve cognitive } \\
\text { function (DMT) }\end{array}$ & Statin & $\begin{array}{l}\text { Adjunct therapy for } \\
\text { myocardial infarction, } \\
\text { revascularization } \\
\text { procedure, angina }\end{array}$ & Cardiovascular & NCT02913664 \\
\hline $\begin{array}{l}\text { AXS-05 (DM+ } \\
\text { Bupropion) }\end{array}$ & II/III & $\begin{array}{l}\text { Neurotransmitter- } \\
\text { based }\end{array}$ & $\begin{array}{l}\text { Improve } \\
\text { neuropsychiatric } \\
\text { symptoms } \\
\text { (agitation) }\end{array}$ & $\begin{array}{l}\text { Norepinephrine- } \\
\text { dopamine } \\
\text { reuptake inhibitor }\end{array}$ & $\begin{array}{l}\text { Major depressive disorder; } \\
\text { easonal affective disorder; } \\
\text { smoking cessation }\end{array}$ & Psychiatric & NCT03226522 \\
\hline Escitalopram & III & $\begin{array}{l}\text { Neurotransmitter- } \\
\text { based }\end{array}$ & $\begin{array}{l}\text { Improve } \\
\text { neuropsychiatric } \\
\text { symptoms } \\
\text { (agitation) }\end{array}$ & $\begin{array}{l}\text { Selective } \\
\text { serotonin } \\
\text { reuptake inhibitor }\end{array}$ & $\begin{array}{l}\text { Major depressive disorder; } \\
\text { generalized anxiety } \\
\text { disorder }\end{array}$ & Psychiatric & NCT03108846 \\
\hline Guanfacine & III & $\begin{array}{l}\text { Neurotransmitter- } \\
\text { based }\end{array}$ & $\begin{array}{l}\text { Modulation of } \\
\text { noradrenergic } \\
\text { deficit (cognitive } \\
\text { enhancer) }\end{array}$ & $\begin{array}{l}\text { Alpha-receptor } \\
\text { agonist }\end{array}$ & $\begin{array}{l}\text { Attention deficit } \\
\text { hyperactivity disorder }\end{array}$ & Cardiovascular & NCT03116126 \\
\hline Icosapent ethyl & II/III & Neuroprotective & $\begin{array}{l}\text { Protect neurons } \\
\text { from disease } \\
\text { pathology (DMT) }\end{array}$ & $\begin{array}{l}\text { Purified w-3 fatty } \\
\text { acid (eicosapenta- } \\
\text { enoic acid) }\end{array}$ & Hypertriglyceridemia & Cardiovascular & NCT02719327 \\
\hline $\begin{array}{l}\text { Levetiracetam } \\
\text { (AGB101) }\end{array}$ & III & Neuroprotective & $\begin{array}{l}\text { Decrease amyloid- } \\
\text { induced neuronal } \\
\text { hyperactivity (DMT) }\end{array}$ & Anticonvulsant & $\begin{array}{l}\text { Adjunct therapy for } \\
\text { partial-onset seizures; ju- } \\
\text { venile myoclonic epilepsy; } \\
\text { primary generalized tonic- } \\
\text { clonic seizures }\end{array}$ & Neurologic & NCT03486938 \\
\hline Losartan & II/III & $\begin{array}{l}\text { Anti- } \\
\text { inflammatory, } \\
\text { metabolic }\end{array}$ & $\begin{array}{l}\text { Vascular risk } \\
\text { reduction to } \\
\text { preserve cognitive } \\
\text { function (DMT) }\end{array}$ & $\begin{array}{l}\text { Angiotensin II } \\
\text { receptor blocker }\end{array}$ & $\begin{array}{l}\text { Hypertension; diabetic } \\
\text { neuropathy; risk reduction } \\
\text { of stroke }\end{array}$ & Cardiovascular & NCT02913664 \\
\hline Masitinib & III & $\begin{array}{l}\text { Anti- } \\
\text { inflammatory }\end{array}$ & $\begin{array}{l}\text { Activity on mast } \\
\text { cells, modulation of } \\
\text { inflammatory } \\
\text { processes (DMT) }\end{array}$ & $\begin{array}{l}\text { Selective tyrosine } \\
\text { kinase inhibitor }\end{array}$ & $\begin{array}{l}\text { Mast cell tumor } \\
\text { (veterinary) }\end{array}$ & $\begin{array}{l}\text { Hematologic- } \\
\text { oncologic }\end{array}$ & $\begin{array}{l}\text { NCT01872598 } \\
(2010-021218- \\
50)\end{array}$ \\
\hline Metformin & II/III & Metabolic & $\begin{array}{l}\text { Improve insulin } \\
\text { sensitivity, may } \\
\text { improve cognition } \\
\text { (DMT) }\end{array}$ & $\begin{array}{l}\text { Insulin sensitizer } \\
\text { (biguanide) }\end{array}$ & Type 2 diabetes & Antidiabetic & NCT04098666 \\
\hline Methylphenidate & III & $\begin{array}{l}\text { Neurotransmitter- } \\
\text { based }\end{array}$ & $\begin{array}{l}\text { Improve } \\
\text { neuropsychiatric } \\
\text { symptoms (apathy) }\end{array}$ & Stimulant & $\begin{array}{l}\text { Attention deficit } \\
\text { hyperactivity disorder }\end{array}$ & Psychiatric & NCT02346201 \\
\hline Mirtazapine & III & $\begin{array}{l}\text { Neurotransmitter- } \\
\text { based }\end{array}$ & $\begin{array}{l}\text { Improve } \\
\text { neuropsychiatric } \\
\text { symptoms } \\
\text { (agitation) }\end{array}$ & $\begin{array}{l}\text { Tetracyclic } \\
\text { antidepressant }\end{array}$ & Major depressive disorder & Psychiatric & NCT03031184 \\
\hline Zolpidem & III & $\begin{array}{l}\text { Neurotransmitter- } \\
\text { based }\end{array}$ & $\begin{array}{l}\text { Improve } \\
\text { neuropsychiatric } \\
\text { symptoms (sleep } \\
\text { disorders) }\end{array}$ & Sedative-Hypnotic & Insomnia & Neurologic & NCT03075241 \\
\hline Zopiclone & III & $\begin{array}{l}\text { Neurotransmitter- } \\
\text { based }\end{array}$ & $\begin{array}{l}\text { Improve } \\
\text { neuropsychiatric }\end{array}$ & Sedative-Hypnotic & Insomnia & Neurologic & NCT03075241 \\
\hline
\end{tabular}


Table 2 Repurposed agents currently in Phase III in the Alzheimer's disease development pipeline (as of February 27, 2020) (Continued)

\begin{tabular}{|c|c|c|c|c|c|c|c|}
\hline Agent name & Phase & $\begin{array}{l}\text { Agent } \\
\text { mechanism class }\end{array}$ & $\begin{array}{l}\text { Therapeutic } \\
\text { purpose under } \\
\text { study }\end{array}$ & Drug class & $\begin{array}{l}\text { FDA-approved } \\
\text { indication(s) }\end{array}$ & $\begin{array}{l}\text { Therapeutic } \\
\text { field }\end{array}$ & $\begin{array}{l}\text { ClinicalTrials.gov } \\
\text { ID(s) (EudraCT } \\
\text { ID) }\end{array}$ \\
\hline & & & $\begin{array}{l}\text { symptoms (sleep } \\
\text { disorders) }\end{array}$ & & & & \\
\hline
\end{tabular}

Abbreviations: DMT disease-modifying therapy, DM dextromethorphan

areas represented included four neurologic drugs, two hematologic-oncologic agents, one drug with pulmonary indications, and one antidiabetic agent.

\section{Discussion}

Drug repurposing provides the unique opportunity to significantly shorten the development period of a therapeutic candidate for AD. These agents have been extensively assessed for pharmacokinetic properties and toxicities normally required in nonclinical and Phase 1 stages of development. Over half of all agents fail in development for these two reasons, and repurposing circumvents these challenges [18]. FDA-approved drugs have already undergone human trials, and safety, efficacy, and adverse event profiles are known. The results of previous trials may suffice to bypass nonclinical stages and earlier clinical phases of study; this has the advantage of foregoing the financial and time-costs associated with a drug development program. Total costs for developing a new agent may be as high as $\$ 5.7$ billion with $\$ 1.66$ billion required for non-clinical studies and \$1.19 billion for Phase I [19]. Total development costs may be reduced by $50 \%$ compared to the cost of developing a new chemical entity.

FDA-approved agents have been optimized for the original indication. Different doses might be needed, and different adverse event profiles may occur in an $\mathrm{AD}$ population compared to other populations for which the agent was originally developed. Some steps in the development process might be repeated to account for these contingencies. Repurposed agents are often generic and have limitations on intellectual property rights that compromise attracting investments that could propel their development [20].

Examination of the repurposed agents in the current AD pipeline revealed that DMT trials were chiefly based on agents derived from hematologic-oncologic (tyrosine kinase inhibitors), cardiovascular (angiotensin II receptor antagonists, angiotensin-converting enzyme inhibitors), and neurologic indications (anticonvulsants), as well as diabetes. Growing evidence supports the association between the key pathologic changes these drugs target and the underlying biology of AD [21-24]. The links between dementia-related symptoms and the abnormal activation of tyrosine kinase pathways, cardiovascular disease, and impaired insulin sensitivity are reflected by the presence of these agents in the pipeline of repurposed drugs. With increasing knowledge of these processes in $\mathrm{AD}$, repurposing may comprise a precision medicine approach to AD therapeutics [25]. A preponderance of psychiatric compounds comprise the symptomatic agent class, and the vast majority (including some non-antipsychotics) are aimed at alleviating ADassociated agitation. This may show a priority in the field to identify treatments for a distressing aspect of $\mathrm{AD}$ for patients and caregivers. These trials were facilitated by the development of new diagnostic criteria for agitation that assist in patient identification and outcome determination [26].

Certain repurposed candidates may offer therapeutic benefits without directly engaging AD targets. Reduced cerebral blood flow correlates with AD pathology [27]. A pilot study has suggested that the antiplatelet agent, cilostazol (NCT02491268), may slow cognitive decline through pleiotropic effects, including regional enhancement of cerebral perfusion [28-30]. Some drugs target the role of neuroinflammation in AD: inflammation affecting the function of the blood-brain barrier is a component of interest in two trials of montelukast (NCT03402503, NCT03991988, [31, 32]). Trialists studying rifaximin (NCT03856359) posit an improvement in cognitive function by reduction in pro-inflammatory cytokines and ammonia levels [32].

Repurposing agent trials make up $39 \%$ of the total AD pipeline in 2020 (compared to $24 \%$ in 2016). Phase II has the largest number of trials-in both repurposing trials and in the overall pipeline (60 and 55\%, respectively). This likely results from entry of repurposed agents directly into Phase II or Phase I/II. DMT trials comprise approximately $79 \%$ of the $2020 \mathrm{AD}$ pipeline, compared to $75 \%$ when looking at the subset of repurposed agents. Although amyloid is the most common specific target in Phase II and Phase III DMT trials overall, the majority of repurposed DMTs involve tau targets.

Forty-six percent of all AD trials are funded at least in part by biopharmaceutical industry sources. In contrast, the majority of repurposed trials $(79 \%)$ receive partial or complete funding from Academic Medical Centers and only 9\% are sponsored by the biopharmaceutical industry. This reflects the challenges of obtaining intellectual property protection for repurposed agents that discourage for-profit enterprises 
Table 3 Repurposed agents currently in Phase II in the Alzheimer's disease development pipeline (as of February 27, 2020)

\begin{tabular}{|c|c|c|c|c|c|c|c|}
\hline Agent name & Phase & $\begin{array}{l}\text { Agent } \\
\text { mechanism class }\end{array}$ & $\begin{array}{l}\text { Therapeutic purpose } \\
\text { under study }\end{array}$ & Drug class & $\begin{array}{l}\text { FDA-approved } \\
\text { indication(s) }\end{array}$ & $\begin{array}{l}\text { Therapeutic } \\
\text { field }\end{array}$ & $\begin{array}{l}\text { ClinicalTrials.gov } \\
\text { ID(s) (EudraCT } \\
\text { ID) }\end{array}$ \\
\hline Benfotiamine & II & Metabolic & $\begin{array}{l}\text { Improve multiple cellular } \\
\text { processes; minimize } \\
\text { decline in glucose } \\
\text { utilization (DMT) }\end{array}$ & $\begin{array}{l}\text { Synthetic } \\
\text { thiamine }\end{array}$ & $\begin{array}{l}\text { Diabetic } \\
\text { nephropathy; type } 2 \\
\text { diabetes }\end{array}$ & Antidiabetic & NCT02292238 \\
\hline Candesartan & II & $\begin{array}{l}\text { Anti-amyloid, } \\
\text { neuroprotective, } \\
\text { metabolic }\end{array}$ & $\begin{array}{l}\text { Improve vascular function } \\
\text { and reduce amyloid (DMT) }\end{array}$ & $\begin{array}{l}\text { Angiotensin II } \\
\text { Receptor Blocker }\end{array}$ & $\begin{array}{l}\text { Hypertension; heart } \\
\text { failure }\end{array}$ & Cardiovascular & NCT02646982 \\
\hline Cilostazol & II & Neuroprotective & $\begin{array}{l}\text { Reduce accumulation of } \\
\text { amyloid and reduce tau } \\
\text { phosphorylation; improve } \\
\text { cerebral circulation (DMT) }\end{array}$ & Antiplatelet & $\begin{array}{l}\text { Intermittent } \\
\text { claudication }\end{array}$ & $\begin{array}{l}\text { Hematologic- } \\
\text { oncologic }\end{array}$ & NCT02491268 \\
\hline Dapagliflozin & $\mathrm{I} / \mathrm{II}$ & Metabolic & $\begin{array}{l}\text { Improve insulin sensitivity } \\
\text { (DMT) }\end{array}$ & $\begin{array}{l}\text { Sodium-glucose } \\
\text { co-transporter } 2 \\
\text { inhibitor }\end{array}$ & $\begin{array}{l}\text { Adjunct therapy for } \\
\text { type } 2 \text { diabetes }\end{array}$ & Antidiabetic & NCT03801642 \\
\hline Daratumumab & II & $\begin{array}{l}\text { Anti- } \\
\text { inflammatory }\end{array}$ & $\begin{array}{l}\text { Broad range } \\
\text { immunomodulatory } \\
\text { effects; regulate microglia } \\
\text { (DMT) }\end{array}$ & $\begin{array}{l}\text { Human antibody } \\
\text { targeting CD38 }\end{array}$ & Multiple myeloma & $\begin{array}{l}\text { Hematologic- } \\
\text { oncologic }\end{array}$ & NCT04070378 \\
\hline $\begin{array}{l}\text { Dasatinib + } \\
\text { Quercetin } \\
\text { (Combo) }\end{array}$ & $\mathrm{I} / \mathrm{II}$ & $\begin{array}{l}\text { Anti- } \\
\text { inflammatory }\end{array}$ & $\begin{array}{l}\text { Senolytic therapy } \\
\text { approach to reduce } \\
\text { senescent cells and tau } \\
\text { aggregation (DMT) }\end{array}$ & $\begin{array}{l}\text { Tyrosine kinase } \\
\text { inhibitor }+ \\
\text { antioxidant/anti- } \\
\text { inflammatory } \\
\text { (Flavonoid) }\end{array}$ & $\begin{array}{l}\text { Philadelphia } \\
\text { chromosome- } \\
\text { positive chronic } \\
\text { myeloid leukemia in } \\
\text { chronic phase }\end{array}$ & $\begin{array}{l}\text { Hematologic- } \\
\text { oncologic }\end{array}$ & NCT04063124 \\
\hline Deferiprone & II & $\begin{array}{l}\text { Anti-Amyloid, } \\
\text { neuroprotective }\end{array}$ & $\begin{array}{l}\text { Reduce reactive oxygen } \\
\text { species that damage } \\
\text { neurons; effect on } \\
\text { amyloid and BACE } \\
\text { pathology (DMT) }\end{array}$ & Iron chelator & $\begin{array}{l}\text { Iron overload } \\
\text { (thalassemia } \\
\text { syndromes) }\end{array}$ & $\begin{array}{l}\text { Hematologic- } \\
\text { oncologic }\end{array}$ & NCT03234686 \\
\hline Dronabinol & II & $\begin{array}{l}\text { Neurotransmitter- } \\
\text { based }\end{array}$ & $\begin{array}{l}\text { Improve neuropsychiatric } \\
\text { symptoms (agitation) }\end{array}$ & Cannabinoid & $\begin{array}{l}\text { Anorexia (associated } \\
\text { with AIDS); nausea/ } \\
\text { vomiting (associated } \\
\text { with chemotherapy) }\end{array}$ & Cannabinoid & NCT02792257 \\
\hline Insulin glulisine & II & Metabolic & $\begin{array}{l}\text { Enhance cell signaling and } \\
\text { growth; promote neuronal } \\
\text { metabolism (DMT) }\end{array}$ & Insulin & Diabetes & Antidiabetic & NCT02503501 \\
\hline Lenolidomide & II & $\begin{array}{l}\text { Anti- } \\
\text { inflammatory }\end{array}$ & $\begin{array}{l}\text { Reduce inflammatory } \\
\text { cytokines (TNF-a, IL-6, IL-8); } \\
\text { reduce inflammatory and } \\
\text { AD-associated biomarkers; } \\
\text { improve cognition (DMT) }\end{array}$ & Anti-neoplastic & Multiple myeloma & $\begin{array}{l}\text { Hematologic- } \\
\text { oncologic }\end{array}$ & NCT04032626 \\
\hline Levetiracetam & II & Neuroprotective & $\begin{array}{l}\text { Reduce amyloid induced } \\
\text { neuronal hyperactivity } \\
\text { (DMT) }\end{array}$ & Anticonvulsant & $\begin{array}{l}\text { Adjunct therapy for } \\
\text { partial-onset seizures; } \\
\text { juvenile myoclonic } \\
\text { epilepsy; primary } \\
\text { generalized tonic- } \\
\text { clonic seizures }\end{array}$ & Neurologic & $\begin{array}{l}\text { NCT03489044 } \\
(2016-003109- \\
32)\end{array}$ \\
\hline Levetiracetam & II & Neuroprotective & $\begin{array}{l}\text { Reduce amyloid induced } \\
\text { neuronal hyperactivity } \\
\text { (DMT) }\end{array}$ & Anticonvulsant & $\begin{array}{l}\text { Adjunct therapy for } \\
\text { partial-onset seizures; } \\
\text { juvenile myoclonic } \\
\text { epilepsy; primary } \\
\text { generalized tonic- } \\
\text { clonic seizures }\end{array}$ & Neurologic & NCT02002819 \\
\hline Levetiracetam & II & Neuroprotective & $\begin{array}{l}\text { Reduce amyloid induced } \\
\text { neuronal hyperactivity } \\
\text { (DMT) }\end{array}$ & Anticonvulsant & $\begin{array}{l}\text { Adjunct therapy for } \\
\text { partial-onset seizures; } \\
\text { juvenile myoclonic } \\
\text { epilepsy; primary } \\
\text { generalized tonic- } \\
\text { clonic seizures }\end{array}$ & Neurologic & NCT03875638 \\
\hline Levetiracetam & II & Neuroprotective & Reduce amyloid induced & Anticonvulsant & Adjunct therapy for & Neurologic & NCT03461861 \\
\hline
\end{tabular}


Table 3 Repurposed agents currently in Phase II in the Alzheimer's disease development pipeline (as of February 27, 2020) (Continued)

\begin{tabular}{|c|c|c|c|c|c|c|c|}
\hline Agent name & Phase & $\begin{array}{l}\text { Agent } \\
\text { mechanism class }\end{array}$ & $\begin{array}{l}\text { Therapeutic purpose } \\
\text { under study }\end{array}$ & Drug class & $\begin{array}{l}\text { FDA-approved } \\
\text { indication(s) }\end{array}$ & $\begin{array}{l}\text { Therapeutic } \\
\text { field }\end{array}$ & $\begin{array}{l}\text { ClinicalTrials.gov } \\
\text { ID(s) (EudraCT } \\
\text { ID) }\end{array}$ \\
\hline (AGB101) & & & $\begin{array}{l}\text { neuronal hyperactivity } \\
\text { (DMT) }\end{array}$ & & $\begin{array}{l}\text { partial-onset seizures; } \\
\text { juvenile myoclonic } \\
\text { epilepsy; primary } \\
\text { generalized tonic- } \\
\text { clonic seizures }\end{array}$ & & \\
\hline Liraglutide & II & Metabolic & $\begin{array}{l}\text { Enhance cell signaling; } \\
\text { improve CNS glucose } \\
\text { metabolism (DMT) }\end{array}$ & $\begin{array}{l}\text { Glucagon-like } \\
\text { peptide-1 agonist }\end{array}$ & $\begin{array}{l}\text { Adjunct therapy for } \\
\text { type } 2 \text { diabetes; risk } \\
\text { reduction of major } \\
\text { cardiovascular events }\end{array}$ & Antidiabetic & $\begin{array}{l}\text { NCT01843075 } \\
(2013-000962- \\
13)\end{array}$ \\
\hline Lithium & II & $\begin{array}{l}\text { Neurotransmitter- } \\
\text { based }\end{array}$ & $\begin{array}{l}\text { Improve neuropsychiatric } \\
\text { symptoms (agitation/ } \\
\text { aggression with or } \\
\text { without psychosis) }\end{array}$ & Antimanic & Bipolar disorder & Psychiatric & NCT02129348 \\
\hline $\begin{array}{l}\text { Leuprolide } \\
\text { depot }\end{array}$ & II & Metabolic & $\begin{array}{l}\text { Alleviates negative effects } \\
\text { of elevated GnRH and } \\
\text { gonadtropins on the brain } \\
\text { (DMT) }\end{array}$ & $\begin{array}{l}\text { Gonadotropin- } \\
\text { releasing } \\
\text { hormone agonist }\end{array}$ & $\begin{array}{l}\text { Endometriosis; } \\
\text { uterine leiomyomata }\end{array}$ & Hormonal & NCT03649724 \\
\hline $\begin{array}{l}\text { Montelukast } \\
\text { (buccal film) }\end{array}$ & II & $\begin{array}{l}\text { Anti- } \\
\text { inflammatory }\end{array}$ & $\begin{array}{l}\text { Reduce inflammatory } \\
\text { pathways (neuronal injury, } \\
\text { blood-brain barrier integ- } \\
\text { rity, amyloid- } B \text { accumula- } \\
\text { tion); effect on cognition } \\
\text { and AD biomarkers (DMT) }\end{array}$ & $\begin{array}{l}\text { Leukotriene } \\
\text { receptor } \\
\text { antagonist }\end{array}$ & $\begin{array}{l}\text { Asthma; exercise- } \\
\text { induced broncho- } \\
\text { constriction; allergic } \\
\text { rhinitis }\end{array}$ & $\begin{array}{l}\text { Anti- } \\
\text { inflammatory }\end{array}$ & NCT03402503 \\
\hline $\begin{array}{l}\text { Montelukast } \\
\text { (tablet) }\end{array}$ & II & $\begin{array}{l}\text { Anti- } \\
\text { inflammatory }\end{array}$ & $\begin{array}{l}\text { Reduce inflammatory } \\
\text { pathways that mediate } \\
\text { neuronal injury, blood- } \\
\text { brain barrier integrity, } \\
\text { amyloid-ß accumulation } \\
\text { (DMT) }\end{array}$ & $\begin{array}{l}\text { Leukotriene } \\
\text { receptor } \\
\text { antagonist }\end{array}$ & $\begin{array}{l}\text { Asthma; exercise- } \\
\text { induced broncho- } \\
\text { constriction; allergic } \\
\text { rhinitis }\end{array}$ & $\begin{array}{l}\text { Anti- } \\
\text { inflammatory }\end{array}$ & NCT03991988 \\
\hline Nicotinamide & II & $\begin{array}{l}\text { Anti-tau, } \\
\text { neuroprotective }\end{array}$ & $\begin{array}{l}\text { Reduce tau-induced } \\
\text { microtubule } \\
\text { depolymerization; reduce } \\
\text { phosphorylation of tau } \\
\text { (DMT) }\end{array}$ & Vitamin B3 & Pellagra & Vitamin & NCT03061474 \\
\hline $\begin{array}{l}\text { Nicotine } \\
\text { (transdermal } \\
\text { patch) }\end{array}$ & II & $\begin{array}{l}\text { Neurotransmitter- } \\
\text { based }\end{array}$ & $\begin{array}{l}\text { Enhance acetylcholine } \\
\text { signaling (cognitive } \\
\text { enhancer) }\end{array}$ & Alkaloid & $\begin{array}{l}\text { Smoking cessation } \\
\text { withdrawal } \\
\text { symptoms }\end{array}$ & Alkaloid & NCT02720445 \\
\hline Nilotinib & II & $\begin{array}{l}\text { Anti-amyloid, } \\
\text { anti-tau }\end{array}$ & $\begin{array}{l}\text { Autophagy enhancer; } \\
\text { promotes clearance of } \\
\text { amyloid and tau proteins } \\
\text { (DMT) }\end{array}$ & $\begin{array}{l}\text { Tyrosine kinase } \\
\text { inhibitor }\end{array}$ & $\begin{array}{l}\text { Philadelphia } \\
\text { chromosome- } \\
\text { positive chronic } \\
\text { myeloid leukemia in } \\
\text { chronic phase }\end{array}$ & $\begin{array}{l}\text { Hematologic- } \\
\text { oncologic }\end{array}$ & NCT02947893 \\
\hline Perindopril & II & $\begin{array}{l}\text { Neuroprotective, } \\
\text { anti-inflammatory }\end{array}$ & $\begin{array}{l}\text { Improves vascular } \\
\text { functioning (DMT) }\end{array}$ & $\begin{array}{l}\text { Angiotensin- } \\
\text { converting- } \\
\text { enzyme inhibitor }\end{array}$ & $\begin{array}{l}\text { Essential } \\
\text { hypertension; stable } \\
\text { coronary artery } \\
\text { disease }\end{array}$ & Cardiovascular & NCT02085265 \\
\hline Prazosin & II & $\begin{array}{l}\text { Neurotransmitter- } \\
\text { based }\end{array}$ & $\begin{array}{l}\text { Improve neuropsychiatric } \\
\text { symptoms (agitation) }\end{array}$ & $\begin{array}{l}\text { Alpha-receptor } \\
\text { antagonist }\end{array}$ & Hypertension & Cardiovascular & NCT03710642 \\
\hline Rifaximin & II & $\begin{array}{l}\text { Anti- } \\
\text { inflammatory }\end{array}$ & $\begin{array}{l}\text { Improve cognition and } \\
\text { function by lowering } \\
\text { blood ammonia; lower } \\
\text { circulatory pro- } \\
\text { inflammatory cytokines se- } \\
\text { creted by harmful gut } \\
\text { bacteria (DMT) }\end{array}$ & $\begin{array}{l}\text { Non-systemic } \\
\text { antibiotic }\end{array}$ & $\begin{array}{l}\text { Traveler's diarrhea; } \\
\text { overt hepatic } \\
\text { encephalopathy; } \\
\text { irritable bowel } \\
\text { syndrome (diarrhea } \\
\text { subtype) }\end{array}$ & $\begin{array}{l}\text { Infectious } \\
\text { disease }\end{array}$ & NCT03856359 \\
\hline Riluzole & II & Neuroprotective & $\begin{array}{l}\text { Reduce glutamate- } \\
\text { mediated excitotoxicity }\end{array}$ & Benzothiazole & $\begin{array}{l}\text { Amyotrophic lateral } \\
\text { sclerosis }\end{array}$ & Neurologic & NCT01703117 \\
\hline
\end{tabular}
(DMT) 
Table 3 Repurposed agents currently in Phase II in the Alzheimer's disease development pipeline (as of February 27, 2020)

\begin{tabular}{|c|c|c|c|c|c|c|c|}
\hline Agent name & Phase & $\begin{array}{l}\text { Agent } \\
\text { mechanism class }\end{array}$ & $\begin{array}{l}\text { Therapeutic purpose } \\
\text { under study }\end{array}$ & Drug class & $\begin{array}{l}\text { FDA-approved } \\
\text { indication(s) }\end{array}$ & $\begin{array}{l}\text { Therapeutic } \\
\text { field }\end{array}$ & $\begin{array}{l}\text { ClinicalTrials.gov } \\
\text { ID(s) (EudraCT } \\
\text { ID) }\end{array}$ \\
\hline Sagramostim & II & $\begin{array}{l}\text { Anti-amyloid, } \\
\text { neuroprotective }\end{array}$ & $\begin{array}{l}\text { Stimulates innate immune } \\
\text { system to remove amyloid } \\
\text { pathology; increase } \\
\text { neuronal connectivity } \\
\text { (DMT) }\end{array}$ & $\begin{array}{l}\text { Human } \\
\text { granulocyte- } \\
\text { macrophage } \\
\text { colony- } \\
\text { stimulating factor }\end{array}$ & $\begin{array}{l}\text { Bone marrow } \\
\text { stimulation }\end{array}$ & $\begin{array}{l}\text { Hematologic- } \\
\text { oncologic }\end{array}$ & NCT01409915 \\
\hline $\begin{array}{l}\text { Sodium } \\
\text { phenylbutyrate }\end{array}$ & II & Neuroprotective & $\begin{array}{l}\text { Blocks nerve cell death } \\
\text { and modulates } \\
\text { neuroinflammation (DMT) }\end{array}$ & $\begin{array}{l}\text { Anti- } \\
\text { hyperammonemia }\end{array}$ & $\begin{array}{l}\text { Adjunct for chronic } \\
\text { urea cycle disorders }\end{array}$ & Genetics & NCT03533257 \\
\hline Tacrolimus & II & Neuroprotective & $\begin{array}{l}\text { Prevents amyloid- } \beta \\
\text { dendritic spine loss; } \\
\text { normalizes cranial nerve } \\
\text { activity in the brain (DMT) }\end{array}$ & $\begin{array}{l}\text { Calcineurin } \\
\text { inhibitor }\end{array}$ & $\begin{array}{l}\text { Prevent rejection of } \\
\text { organ transplants }\end{array}$ & Immunologic & NCT04263519 \\
\hline Telmisartan & II & $\begin{array}{l}\text { Neuroprotective, } \\
\text { anti-inflammatory }\end{array}$ & $\begin{array}{l}\text { Improves vascular } \\
\text { functioning (DMT) }\end{array}$ & $\begin{array}{l}\text { Angiotensin II } \\
\text { Receptor Blocker }\end{array}$ & $\begin{array}{l}\text { Hypertension; } \\
\text { diabetic neuropathy; } \\
\text { risk reduction of } \\
\text { stroke }\end{array}$ & Cardiovascular & NCT02085265 \\
\hline Thiethylperazine & II & Anti-amyloid & $\begin{array}{l}\text { Activates transport protein } \\
\text { ABCC1 and enhances } \\
\text { transport amyloid- } \beta \\
\text { peptides from the brain } \\
\text { into the blood (DMT) }\end{array}$ & Phenothiazine & Nausea/vomiting & Neurologic & $\begin{array}{l}\text { NCT03417986 } \\
(2014-000870- \\
20)\end{array}$ \\
\hline Valacyclovir & II & $\begin{array}{l}\text { Neuroprotective, } \\
\text { anti-inflammatory }\end{array}$ & $\begin{array}{l}\text { Reduce amyloid- } \beta \\
\text { aggregation by } \\
\text { preventing } \\
\text { overproduction of amyloid } \\
\text { in response to infection } \\
\text { (DMT) }\end{array}$ & $\begin{array}{l}\text { Antiviral } \\
\text { (nucleoside } \\
\text { analog) }\end{array}$ & $\begin{array}{l}\text { Herpes labialis; } \\
\text { genital herpes }\end{array}$ & $\begin{array}{l}\text { Infectious } \\
\text { disease }\end{array}$ & NCT03282916 \\
\hline Valacyclovir & II & $\begin{array}{l}\text { Neuroprotective, } \\
\text { anti-inflammatory }\end{array}$ & $\begin{array}{l}\text { Reduce amyloid- } \beta \\
\text { aggregation by } \\
\text { preventing } \\
\text { overproduction of amyloid } \\
\text { in response to infection } \\
\text { (DMT) }\end{array}$ & $\begin{array}{l}\text { Antiviral } \\
\text { (nucleoside } \\
\text { analog) }\end{array}$ & $\begin{array}{l}\text { Herpes labialis; } \\
\text { genital herpes }\end{array}$ & $\begin{array}{l}\text { Infectious } \\
\text { disease }\end{array}$ & NCT02997982 \\
\hline
\end{tabular}

from exercising this development pathway. The observation emphasizes the importance of Academic Medical Centers and federal and philanthropic funding in the $\mathrm{AD}$ drug development ecosystem.

A variety of strategies are being assessed to try to secure intellectual property positions for repurposed agents. Using different doses relative to an agent's original indication is one approach and depends on the drug exposure required for each indication. This strategy is exemplified by the HOPE4MCI study (NCT03486938), which is using one-tenth of the dose of levetiracetam used for anticonvulsant purposes. Another approach is the use of novel formulations of an agent. Insulin glulisine (NCT02503501) is a rapidacting version of the insulin; a long-acting form (NCT01595646) was developed for trials in AD [33]. Montelukast is being investigated as a cognitive enhancer in two separate trials (NCT03991988, NCT03402503) using an oral tablet and a buccal film patch, respectively. This highlights the opportunity to develop novel delivery mechanisms for repurposed agents as a means of enhancing efficacy and securing intellectual property rights.

The marked increase in repurposing may reflect the recent efforts aimed at elucidating the benefits of repurposing as a strategy in $\mathrm{AD}$ drug development [7, 34]. Funding from federal, advocacy, and philanthropic sources disproportionately support trials of repurposed agents and this may have driven the increase in repurposing. Repurposing may have been accelerated by advances in methodologies that are being used to identify AD-efficacious pharmaceuticals that include phenotypic or high content screening, in silico techniques, bioinformatic or computational strategies, and medical geneticbased approaches [9, 35-39]. Electronic health records may provide evidence of beneficial effects on $\mathrm{AD}$ or other neurodegenerative disorders by therapies approved for other indications and represent another means for discovering repurposing agents [39, 40]. The effects of long-term exposure to these agents, however, may not 
Table 4 Repurposed agents currently in Phase I in the Alzheimer's disease development pipeline (as of February 27, 2020)

\begin{tabular}{|c|c|c|c|c|c|c|c|}
\hline Agent name & Phase & $\begin{array}{l}\text { Agent } \\
\text { mechanism class }\end{array}$ & $\begin{array}{l}\text { Therapeutic purpose } \\
\text { under study }\end{array}$ & Drug class & $\begin{array}{l}\text { FDA-approved } \\
\text { indication(s) }\end{array}$ & $\begin{array}{l}\text { Therapeutic } \\
\text { field }\end{array}$ & $\begin{array}{l}\text { ClinicalTrials.gov } \\
\operatorname{ID}(s)\end{array}$ \\
\hline $\begin{array}{l}\text { Allopregnanolone } \\
\text { (Brexanolone) }\end{array}$ & I & $\begin{array}{l}\text { Neuroprotective, } \\
\text { metabolic }\end{array}$ & $\begin{array}{l}\text { Enhanced neurogenesis } \\
\text { slow hippocampal } \\
\text { atrophy; improve } \\
\text { synaptic connectivity } \\
\text { (DMT) }\end{array}$ & $\begin{array}{l}\text { GABA-A } \\
\text { receptor } \\
\text { modulator; } \\
\text { neuroactive } \\
\text { steroid } \\
\text { (antidepressant) }\end{array}$ & $\begin{array}{l}\text { Postpartum } \\
\text { depression }\end{array}$ & Psychiatric & NCT03748303 \\
\hline $\begin{array}{l}\text { BDPP (Bioactive } \\
\text { Dietary } \\
\text { Polyphenol } \\
\text { Preparation) }\end{array}$ & 1 & Neuroprotective & $\begin{array}{l}\text { Prevents amyloid and } \\
\text { tau aggregation (DMT) }\end{array}$ & Polyphenol & Dietary supplement & Nutraceutical & NCT02502253 \\
\hline Dabigatran & I & Neuroprotective & $\begin{array}{l}\text { Reduce neurovascular } \\
\text { damage (DMT) }\end{array}$ & Anticoagulant & $\begin{array}{l}\text { Reduce risk of stroke; } \\
\text { embolism associated } \\
\text { with atrial fibrillation, } \\
\text { deep venous } \\
\text { thrombosis, } \\
\text { pulmonary embolism }\end{array}$ & $\begin{array}{l}\text { Hematologic- } \\
\text { oncologic }\end{array}$ & NCT03752294 \\
\hline Efavirenz & 1 & Anti-amyloid & $\begin{array}{l}\text { Increase cholesterol } \\
\text { removal and enhance } \\
\text { amyloid reduction (DMT) }\end{array}$ & $\begin{array}{l}\text { Non-nucleoside } \\
\text { reverse- } \\
\text { transcriptase } \\
\text { inhibitor }\end{array}$ & HIV/AIDS & $\begin{array}{l}\text { Infectious } \\
\text { disease }\end{array}$ & NCT03706885 \\
\hline Escitalopram & 1 & $\begin{array}{l}\text { Neurotransmitter- } \\
\text { based }\end{array}$ & $\begin{array}{l}\text { Increase } \\
\text { neurotransmission } \\
\text { (cognitive enhancer) }\end{array}$ & $\begin{array}{l}\text { Selective } \\
\text { serotonin } \\
\text { reuptake } \\
\text { inhibitor }\end{array}$ & $\begin{array}{l}\text { Major depressive } \\
\text { disorder; generalized } \\
\text { anxiety disorder }\end{array}$ & Psychiatric & NCT03274817 \\
\hline Empagliflozin & 1 & Metabolic & $\begin{array}{l}\text { Increase ketone levels in } \\
\text { the brain; improve } \\
\text { neuronal health and } \\
\text { delay onset and } \\
\text { progression of cognitive } \\
\text { impairment (DMT) }\end{array}$ & $\begin{array}{l}\text { Sodium-glucose } \\
\text { co-transporter } 2 \\
\text { inhibitor }\end{array}$ & $\begin{array}{l}\text { Adjunct therapy for } \\
\text { type } 2 \text { diabetes }\end{array}$ & Antidiabetic & NCT03852901 \\
\hline Salsalate & I & $\begin{array}{l}\text { Anti- } \\
\text { inflammatory }\end{array}$ & $\begin{array}{l}\text { Reduce neuronal injury } \\
\text { (DMT) }\end{array}$ & $\begin{array}{l}\text { Non-steroidal } \\
\text { anti- } \\
\text { inflammatory } \\
\text { drug }\end{array}$ & $\begin{array}{l}\text { Rheumatoid arthritis; } \\
\text { osteoarthritis }\end{array}$ & $\begin{array}{l}\text { Anti- } \\
\text { inflammatory }\end{array}$ & NCT03277573 \\
\hline Telmisartan & I & $\begin{array}{l}\text { Neuroprotective, } \\
\text { anti-inflammatory }\end{array}$ & $\begin{array}{l}\text { Improve vascular } \\
\text { functioning and effects } \\
\text { on amyloid pathology } \\
\text { (DMT) }\end{array}$ & $\begin{array}{l}\text { Angiotensin II } \\
\text { Receptor } \\
\text { Blocker }\end{array}$ & $\begin{array}{l}\text { Hypertension; diabetic } \\
\text { neuropathy; risk } \\
\text { reduction of stroke }\end{array}$ & Cardiovascular & NCT02471833 \\
\hline Venlafaxine & 1 & $\begin{array}{l}\text { Neurotransmitter- } \\
\text { based }\end{array}$ & $\begin{array}{l}\text { Increase } \\
\text { neurotransmission } \\
\text { (cognitive enhancer) }\end{array}$ & $\begin{array}{l}\text { Serotonin- } \\
\text { norepinephrine } \\
\text { reuptake } \\
\text { inhibitor }\end{array}$ & $\begin{array}{l}\text { Major depressive } \\
\text { disorder; general } \\
\text { anxiety disorder; } \\
\text { seasonal affective } \\
\text { disorder; panic } \\
\text { disorder }\end{array}$ & Psychiatric & NCT03274817 \\
\hline Vorinostat & I & Neuroprotective & $\begin{array}{l}\text { Multiple cellular } \\
\text { processes including tau } \\
\text { aggregation and } \\
\text { amyloid deposition } \\
\text { (DMT) }\end{array}$ & $\begin{array}{l}\text { Histone } \\
\text { deacetylase } \\
\text { inhibitor } \\
\text { (anticancer) }\end{array}$ & $\begin{array}{l}\text { Cutaneous T-cell } \\
\text { lymphoma }\end{array}$ & $\begin{array}{l}\text { Hematologic- } \\
\text { oncologic }\end{array}$ & NCT03056495 \\
\hline
\end{tabular}

Abbreviations: DMT, disease modifying therapy

be recapitulated in the 18-24 months of most current AD trials.

Between 2016 and 2020, 20 trials of repurposed agents reached a "completed" status while four were "terminated" for various reasons. Post hoc analysis of a pilot Phase II Metformin study (NCT01965756) found the agent to be safe and well-tolerated, with improvements in executive function [41]. In another study, a short course of NewGam 10\% IVIG (intravenous immunoglobulin; NCT01300728) given during the mild cognitive impairment (MCI) stage of AD decreased brain atrophy and ameliorated cognitive decline, but beneficial effects did not persist after 2 years [42]. A phase I study (NCT01780519) found that lorazepam may unmask presymptomatic cognitive dysfunction in individuals with underlying genetic risk for $\mathrm{AD}$ [43]. Primary outcome 
Table 5 Trial sponsor for repurposed agents by phase of drug development (as of February 27, 2020)

\begin{tabular}{|c|c|c|c|c|}
\hline Sponsor & Phase I & Phase II & Phase III & Total (\%) \\
\hline Academic Medical Centers & 6 & 14 & 4 & $24(45.3)$ \\
\hline Biopharma & 0 & 2 & 3 & $5(9.4)$ \\
\hline NIH & 1 & 0 & 0 & $1(1.9)$ \\
\hline NIH and Academic Medical Centers & 0 & 4 & 2 & $6(11.3)$ \\
\hline NIH and consortium & 0 & 1 & 0 & $1(1.9)$ \\
\hline NIH and Industry & 0 & 0 & 1 & $1(1.9)$ \\
\hline Academic Medical Centers and Industry & 0 & 2 & 0 & $2(3.8)$ \\
\hline Academic Medical Centers and Foundation & 1 & 4 & 0 & $5(9.4)$ \\
\hline Academic Medical Centers, Industry, and NIH & 0 & 0 & 1 & $1(1.9)$ \\
\hline Other combinations & 1 & 4 & 0 & $5(9.4)$ \\
\hline Other federal sources & 0 & 1 & 1 & $2(3.8)$ \\
\hline Total & 9 & 32 & 12 & $53(100.0)$ \\
\hline
\end{tabular}

Abbreviations: NIH National Institutes of Health

analyses of the NILVAD study (NCT02017340) failed to show any treatment benefit with nilvadipine when compared to the matched placebo population, though the drug was found to be safe and well-tolerated [44]. The AMBAR study by Grifols (NCT01561053) offers a novel approach to potential AD treatment via therapeutic albumin replacement with or without immunoglobulin [45]. Outcome analyses revealed a statistically significant $61 \%$ decrease in progression of moderate AD who received treatment. None of the trial "failures" results from problems associated with safety or tolerability, which may be indicative of the extensive studies repurposed agents have already undergone.

A single repurposed compound may target different outcomes; escitalopram is being studied simultaneously in trials for agitation and for improvements in cognition (NCT03108846, NCT03274817). Combinations of FDAapproved drugs represent another approach to repurposing; ALZT-OP1 (NCT02482324) uses a two-part combination of repurposed agents-a mast-cell stabilizer (cromolyn) and the non-steroidal anti-inflammatory agent ibuprofen. A combination of losartan, amlodipine, and atorvastatin is a combination trial (NCT02913664) involving repurposed agents to address the vascular dimensions of AD.

We assessed repurposing in the EUCTR and compared it to the analyses of ClinicalTrials.gov. Using the same criteria used to query the US governmental database, we found that repurposing trials make up approximately $10 \%$ of all ongoing EU/EEA AD trials (in contrast to $39 \%$ in the US AD pipeline). Similar to the US pipeline, the majority of trials (64\%) are in Phase II and involve DMT agents (43\%). The majority of agents identified in the EU/EEA trials were among the agents identified in ClinicalTrials.gov; only three trials were unique to the EUCTR.

Some major discrepancies were encountered when comparing both trial registers. Six of the 14 EUCTR trials, or $43 \%$, were found to have a "completed" or "terminated" status on ClinicalTrials.gov (Table 7). Inaccurate reporting of dual-registered trials on EUCTR is a prevalent issue [46], which may in part reflect the register's reliance on sponsors to manually enter study results onto the EudraCT database. Suboptimal completion of RCT protocols and results may lead to overestimates of trial activity [47-50].

Table 6 Repurposing agent trials between 2016 and 2020

\begin{tabular}{|c|c|c|c|c|c|c|c|c|c|c|}
\hline & \multicolumn{2}{|l|}{2016} & \multicolumn{2}{|l|}{2017} & \multicolumn{2}{|l|}{2018} & \multicolumn{2}{|l|}{2019} & \multicolumn{2}{|l|}{2020} \\
\hline & DMTs & $\begin{array}{l}\text { Symptomatic } \\
\text { agents }\end{array}$ & DMTs & $\begin{array}{l}\text { Symptomatic } \\
\text { agents }\end{array}$ & DMTs & $\begin{array}{l}\text { Symptomatic } \\
\text { agents }\end{array}$ & DMTs & $\begin{array}{l}\text { Symptomatic } \\
\text { agents }\end{array}$ & DMTs & $\begin{array}{l}\text { Symptomatic } \\
\text { agents }\end{array}$ \\
\hline Phase I & 1 & 3 & 1 & 2 & 3 & 2 & 7 & 1 & 8 & 1 \\
\hline Phase II & 10 & 7 & 11 & 9 & 15 & 10 & 19 & 7 & 28 & 4 \\
\hline $\begin{array}{l}\text { Phase } \\
\text { III }\end{array}$ & 4 & 3 & 4 & 4 & 2 & 5 & 3 & 6 & 6 & 6 \\
\hline Total & 15 & 13 & 16 & 15 & 20 & 17 & 29 & 14 & 42 & 11 \\
\hline
\end{tabular}

Abbreviations: DMT disease-modifying therapy 


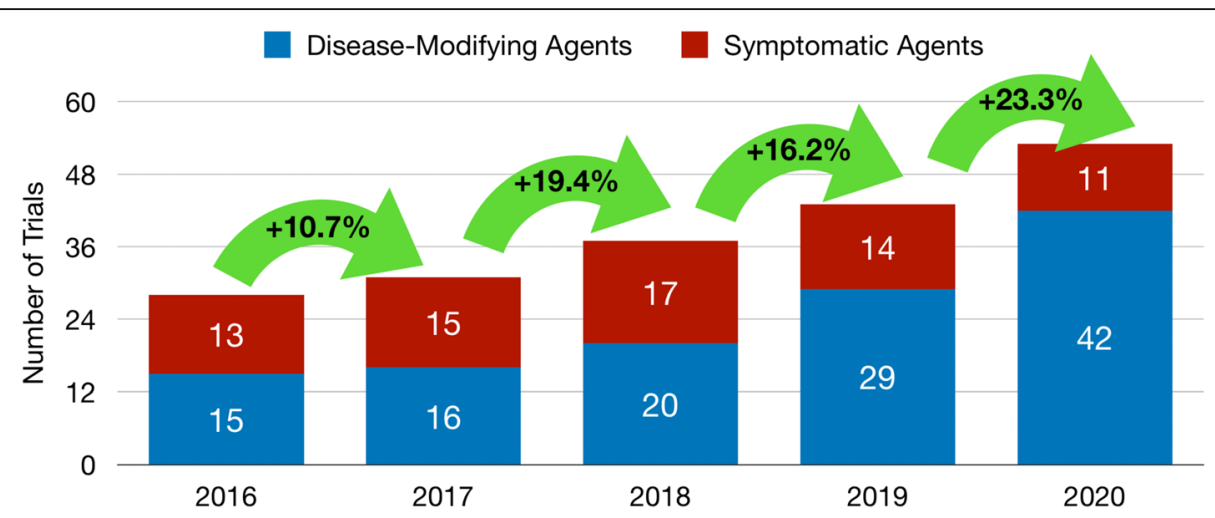

Fig. 4 Repurposing trials over time in the Alzheimer's disease drug development pipeline (2016-2020)

Repurposing is to be distinguished from developing extended indications for a proprietary agent as part of life-cycle management. Pimavanserin, for example, is approved for the treatment of hallucinations and delusions in Parkinson's disease psychosis; an extended application for dementia-related psychosis is currently being developed for several dementia syndromes [51, 52]. Likewise, suvorexant is approved for insomnia and completed a successful trial for insomnia in $\mathrm{AD}$ and the labeling of the compound has been adjusted to include this information. Brexpiprazole is approved for the treatment of schizophrenia and as adjunctive therapy for major depressive disorder; it is being assessed in the $\mathrm{AD}$ pipeline as a therapy for agitation [53]. These programs represent extensions of indications and not repurposing.

Repurposing can be distinguished from repositioning in which a non-approved agent in a sponsor's development program changes from one indication to another because disease-related, compound-related, or commercial circumstances suggest that success in further development may be more likely in one condition than another $[54,55]$.

\section{Limitations}

There are limitations of the current analyses. Because not all Phase I trial activity may be recorded in the clinicaltrials.gov database, our data may underestimate the actual number of trials in this phase. Estimates of drugs designated as DMT versus symptomatic agents may be equivocal depending on what is perceived as an agent's principle MOA. We stopped entering new data into our database at a time that allowed submission, peer review, and publication (final data collection stopped on February 27,2020 ) and there may be newer trials or changes to current trials not reflected in this document.

\section{Conclusions}

In summary, repurposing is a promising avenue for $\mathrm{AD}$ drug development, comprises a substantial part of the current AD pipeline, and is increasing in absolute numbers of agents and as a proportion of the pipeline. Repurposing may reduce drug development costs by up to $50 \%$ and accelerate times lines for progression in the pipeline. Repurposing draws on development programs from numerous other indications. Repurposing is an important part of the current $\mathrm{AD}$ drug development ecosystem.

\section{Abbreviations \\ AD: Alzheimer's disease; AMBAR: Alzheimer's Management by Albumin Replacement; DMT: Disease-modifying therapy; FDA: Food and Drug Administration; IVIG: Intravenous immunoglobulin; MCl: Mild cognitive impairment; MOA: Mechanism of action; NILVAD: Nilvadipine to Treat Alzheimer's Disease}

\section{Acknowledgements \\ Not applicable.}

\section{Authors' contributions}

GL obtained and organized the data regarding trial activity, as well as contributed to the manuscript. JB analyzed the data and was a major contributor in writing the manuscript. All authors read and approved the final manuscript.

\section{Funding}

Not applicable.

\section{Availability of data and materials}

The datasets used and/or analyzed during the current study are available from the corresponding author on reasonable request.

Ethics approval and consent to participate Not applicable.

\section{Consent for publication}

Not applicable.

\section{Competing interests}

$J \mathrm{~L}$ has provided consultation to Acadia, Actinogen, AgeneBio, Alkahest, Alzheon, Annovis, Avanir, Axsome, Biogen, Bracket, Cassava, Cerecin, Cerevel, Cognoptix, Cortexyme, Cytox, EIP Pharma, Eisai, Foresight, Green Valley, Grifols, Hisun, Karuna, Novo Nordisk, Nutricia, Otsuka, ReMYND, Resverlogix, Roche, Samumed, Samus, Signant Health, Sunovion, Suven, Third Rock, and United Neuroscience pharmaceutical and assessment companies. JL has stock options in ADAMAS, MedAvante, QR pharma, BiOasis, and United Neuroscience. JL owns the copyright of the Neuropsychiatric Inventory. Dr. Cummings is supported by Keep Memory Alive (KMA); NIGMS grant 
Table 7 Repurposing agents currently in the European Alzheimer's Disease development pipeline (as of February 27, 2020)

\begin{tabular}{|c|c|c|c|c|c|c|c|}
\hline Agent name & Phase(s) & $\begin{array}{l}\text { Agent } \\
\text { mechanism class }\end{array}$ & $\begin{array}{l}\text { Therapeutic purpose } \\
\text { under study }\end{array}$ & Drug class & $\begin{array}{l}\text { FDA-approved } \\
\text { indication(s) }\end{array}$ & $\begin{array}{l}\text { Therapeutic } \\
\text { field }\end{array}$ & $\begin{array}{l}\text { EudraCT ID } \\
\text { (Clinicaltrials.gov } \\
\text { ID) }\end{array}$ \\
\hline $\begin{array}{l}\text { ALZT-OP1a, } \\
\text { ALZT-OP1b } \\
\text { (cromolyn with } \\
\text { and without } \\
\text { ibuprofen) }\end{array}$ & III & $\begin{array}{l}\text { Anti-amyloid, } \\
\text { anti-inflammatory }\end{array}$ & $\begin{array}{l}\text { Reduce neuronal } \\
\text { damage; mast cells may } \\
\text { also play a role in } \\
\text { amyloid pathology } \\
\text { (DMT) }\end{array}$ & $\begin{array}{l}\text { Mast cell } \\
\text { stabilizer }\end{array}$ & $\begin{array}{l}\text { Bronchial asthma; } \\
\text { mastocytosis }\end{array}$ & Pulmonary & $\begin{array}{l}\text { 2015-002147-34 } \\
\text { (NCT02547818) }\end{array}$ \\
\hline Cannabidiol & II & $\begin{array}{l}\text { Neurotransmitter- } \\
\text { based }\end{array}$ & $\begin{array}{l}\text { Improve } \\
\text { neuropsychiatric } \\
\text { symptoms (agitation) }\end{array}$ & Cannabinoid & $\begin{array}{l}\text { Seizures (Lennox- } \\
\text { Gastaut syndrome or } \\
\text { Dravet syndrome) }\end{array}$ & Neurologic & 2019-002106-52 \\
\hline Dronabinol & II & $\begin{array}{l}\text { Neurotransmitter- } \\
\text { based }\end{array}$ & $\begin{array}{l}\text { Improve } \\
\text { neuropsychiatric } \\
\text { symptoms (agitation) }\end{array}$ & $\begin{array}{l}\text { Synthetic delta-9- } \\
\text { THC }\end{array}$ & $\begin{array}{l}\text { AIDS-associated } \\
\text { anorexia; antiemetic } \\
\text { for chemotherapy- } \\
\text { induced nausea and } \\
\text { vomiting }\end{array}$ & Neurologic & $\begin{array}{l}\text { 2011-005289- } \\
\text { 39* } \\
\text { (NCT01608217) } \\
\text { 2010-024577- } \\
\text { 39* }^{*} \\
\text { (NCT01302340) }\end{array}$ \\
\hline Epoetin alfa & II & $\begin{array}{l}\text { Anti- } \\
\text { inflammatory, } \\
\text { metabolic }\end{array}$ & $\begin{array}{l}\text { Reduce neuronal injury } \\
\text { (DMT); improve } \\
\text { neuropsychiatric } \\
\text { symptoms (depression) }\end{array}$ & $\begin{array}{l}\text { Erythropoiesis- } \\
\text { stimulating } \\
\text { agent }\end{array}$ & $\begin{array}{l}\text { Anemia (chronic } \\
\text { kidney disease, } \\
\text { zidovudine treatment, } \\
\text { chemotherapy); } \\
\text { reduction of red blood } \\
\text { cell transfusion }\end{array}$ & $\begin{array}{l}\text { Hematologic- } \\
\text { oncologic }\end{array}$ & 2008-000453-35 \\
\hline Levetiracetam & II & Neuroprotective & $\begin{array}{l}\text { Reduce amyloid } \\
\text { induced neuronal } \\
\text { hyperactivity (DMT) }\end{array}$ & Anticonvulsant & $\begin{array}{l}\text { Adjunct therapy for } \\
\text { partial-onset seizures; } \\
\text { juvenile myoclonic epi- } \\
\text { lepsy; primary general- } \\
\text { ized tonic-clonic } \\
\text { seizures }\end{array}$ & Neurologic & $\begin{array}{l}\text { 2016-003109-32 } \\
\text { (NCT03489044) }\end{array}$ \\
\hline $\begin{array}{l}\text { Levodopa- } \\
\text { Carbidopa }\end{array}$ & II & $\begin{array}{l}\text { Neurotransmitter- } \\
\text { based }\end{array}$ & $\begin{array}{l}\text { Enhance dopamine } \\
\text { neurotransmission } \\
\text { (cognitive enhancer) }\end{array}$ & $\begin{array}{l}\text { Dopamine } \\
\text { prodrug }\end{array}$ & Parkinson's disease & Neurologic & 2009-011093-15 \\
\hline Liraglutide & II & Metabolic & $\begin{array}{l}\text { Enhance cell signaling; } \\
\text { improve CNS glucose } \\
\text { metabolism (DMT) }\end{array}$ & $\begin{array}{l}\text { Glucagon-like } \\
\text { peptide-1 } \\
\text { agonist }\end{array}$ & $\begin{array}{l}\text { Adjunct therapy for } \\
\text { type } 2 \text { diabetes; risk } \\
\text { reduction of major } \\
\text { cardiovascular events }\end{array}$ & Antidiabetic & $\begin{array}{l}\text { 2013-000962-13 } \\
\text { (NCT01843075) }\end{array}$ \\
\hline Masitinib & III & $\begin{array}{l}\text { Anti- } \\
\text { inflammatory }\end{array}$ & $\begin{array}{l}\text { Activity on mast cells, } \\
\text { modulation of } \\
\text { inflammatory processes } \\
\text { (DMT) }\end{array}$ & $\begin{array}{l}\text { Selective tyrosine } \\
\text { kinase inhibitor }\end{array}$ & $\begin{array}{l}\text { Mast cell tumor } \\
\text { (veterinary) }\end{array}$ & $\begin{array}{l}\text { Hematologic- } \\
\text { oncologic }\end{array}$ & $\begin{array}{l}\text { 2010-021218-50 } \\
\text { (NCT01872598) }\end{array}$ \\
\hline Rosiglitazone & III & Metabolic & $\begin{array}{l}\text { PPAR-gamma agonist } \\
\text { (cognitive enhancer) }\end{array}$ & Thiazolidinedione & $\begin{array}{l}\text { Adjunct therapy for } \\
\text { type } 2 \text { diabetes }\end{array}$ & Antidiabetic & $\begin{array}{l}\mathbf{2 0 1 2 - 0 0 2 7 6 4 -} \\
\mathbf{2 7 * *} \\
\text { (NCTO0550420) } \\
\mathbf{2 0 0 6 - 0 0 1 4 0 3 -} \\
\mathbf{1 1} \mathbf{1}^{*} \\
(\mathrm{NCT} 00348309) \\
\mathbf{2 0 0 6 - 0 0 1 4 0 2 -} \\
\mathbf{9 2} \\
\text { (NCTO0348140) }\end{array}$ \\
\hline Rotigotine & II & $\begin{array}{l}\text { Neurotransmitter- } \\
\text { based }\end{array}$ & $\begin{array}{l}\text { Enhance dopamine } \\
\text { neurotransmission } \\
\text { (cognitive enhancer) }\end{array}$ & $\begin{array}{l}\text { Non-ergoline } \\
\text { dopamine } \\
\text { agonist }\end{array}$ & $\begin{array}{l}\text { Parkinson's disease; } \\
\text { restless leg syndrome }\end{array}$ & Neurologic & $\begin{array}{l}\text { 2015-002965- } \\
\mathbf{4 3}^{*} \\
\text { (NCT03250741) }\end{array}$ \\
\hline Thiethylperazine & II & Anti-amyloid & $\begin{array}{l}\text { Activates transport } \\
\text { protein } A B C C 1 \text { and } \\
\text { enhances transport } \\
\text { amyloid- } \beta \text { peptides } \\
\text { from the brain into the } \\
\text { blood (DMT) }\end{array}$ & Phenothiazine & Nausea/vomiting & Neurologic & $\begin{array}{l}\text { 2014-000870-20 } \\
\text { (NCT03417986) }\end{array}$ \\
\hline
\end{tabular}


P20GM109025; NINDS grant U01NS093334; and NIA grant R01AG053798. JB and $\mathrm{GL}$ have no disclosures.

\section{Author details}

'School of Medicine, University of Nevada, Las Vegas (UNLV), Las Vegas, NV 89154, USA. ²Cleveland Clinic Lou Ruvo Center for Brain Health, Las Vegas, NV 89106, USA. ${ }^{3}$ Chambers-Grundy Center for Transformative Neuroscience, Department of Brain Health, School of Integrated Health Sciences, University of Nevada, Las Vegas (UNLV), Las Vegas, NV 89154, USA.

\section{Received: 28 May 2020 Accepted: 29 July 2020}

\section{Published online: 17 August 2020}

\section{References}

1. Masters CL, Bateman R, Blennow K, Rowe CC, Sperling RA, Cummings JL. Alzheimer's disease. Nat Rev Dis Primers. 2015;1:15056. https://doi.org/10. 1038/nrdp.2015.56.

2. Brookmeyer R, Johnson E, Ziegler-Graham $K$, Arrighi HM. Forecasting the global burden of Alzheimer's disease. Alzheimers Dement. 2007;3(3):186-91. https://doi.org/10.1016/j.jalz.2007.04.381.

3. Aisen P, Schneider L, Cummings J. Sympotmatic and non-amyloid/tau based pharmacologic treatment for Alzheimer's disease. In: Selkoe D, Mandlekow E, Holtzman D, editors. The Biology of Alzheimer's Disease. New York: Cold Springs Harbor Laboratory Press; 2012. p. 511.

4. Boguski MS, Mandl KD, Sukhatme VP. Drug discovery. Repurposing with a difference. Science. 2009;324(5933):1394-5. https://doi.org/10.1126/science. 1169920.

5. Appleby BS, Nacopoulos D, Milano N, Zhong K, Cummings JL. A review: treatment of Alzheimer's disease discovered in repurposed agents. Dement Geriatr Cogn Disord. 2013;35(1-2):1-22. https://doi.org/10.1159/000345791.

6. Corbett A, Williams G, Ballard C. Drug repositioning: an opportunity to develop novel treatments for Alzheimer's disease. Pharmaceuticals (Basel). 2013;6(10):1304-21. https://doi.org/10.3390/ph6101304.

7. Parsons CG. CNS repurposing - potential new uses for old drugs: examples of screens for Alzheimer's disease, Parkinson's disease and spasticity. Neuropharmacology. 2019;147:4-10.

8. Monacelli F, Cea M, Borghi R, Odetti P, Nencioni A. Do cancer drugs counteract neurodegeneration? Repurposing for Alzheimer's disease. Alzheimers Dis. 2017:55:1295-306. https://doi.org/10.3233/JAD-160840.

9. Siavelis JC, Bourdakou MM, Athanasiadis El, Spyrou GM, Nikita KS. Bioinformatics methods in drug repurposing for Alzheimer's disease. Brief Bioinform. 2016;17(2):322-35. https://doi.org/10.1093/bib/bbv048.

10. Cummings J, Morstorf T, Lee G. Alzheimer's drug-development pipeline: 2016. Alzheimers Dement (N Y). 2016;2(4):222-32. https://doi.org/10.1016/j. trci.2016.07.001.

11. Cummings J, Lee G, Mortsdorf T, Ritter A, Zhong K. Alzheimer's disease drug development pipeline: 2017. Alzheimers Dement (N Y). 2017;3(3):367-84. https://doi.org/10.1016/j.trci.2017.05.002

12. Cummings J, Lee G, Ritter A, Zhong K. Alzheimer's disease drug development pipeline: 2018. Alzheimers Dement. 2018;4:195-214. https:// doi.org/10.1016/j.trci.2018.03.009.

13. Cummings J, Lee G, Ritter A, Sabbagh M, Zhong K. Alzheimer's disease drug development pipeline: 2019. Alzheimers Dement (N Y). 2019;5:272-93. https://doi.org/10.1016/j.trci.2019.05.008

14. Cummings J, Lee G, Ritter A, Sabbagh M, Zhong K. Alzheimer's disease drug development pipeline: Alz \& Dem: TRCI 2020; 2020. https://doi.org/10.1002/trc2. 12050

15. Lassman SM, Shopshear OM, Jazic I, Ulrich J, Francer J. Clinical trial transparency: a reassessment of industry compliance with clinical trial registration and reporting requirements in the United States. BMJ Open. 2017;7:e015110.

16. Anderson ML, Chiswell K, Peterson ED, Tasneem A, Topping J, Califf RM. Compliance with results reporting at ClinicalTrials.gov. N Engl J Med. 2015;372:10319

17. Center for Drug Evaluation and Research. Drugs@FDA. U.S. Food and Drug Administration, Washington, DC. 2019. https://www.accessdata.fda.gov/ scripts/cder/daf/index.cfm. 2019. Accessed 15 Mar 2020.

18. Hp R. Drug discovery and development. Edinburgh: Churchill Livingstone; 2006.

19. Scott TJ, O'Connor AC, Link AN, Beaulieu TJ. Economic analysis of opportunities to accelerate Alzheimer's disease research and development. Ann N Y Acad Sci. 2014;1313:17-34.
20. Verbaanderd C, Rooman I, Meheus L, Huys I. On-label or off-label? Overcoming regulatory and financial barriers to bring repurposed medicines to cancer patients. Front Pharmacol. 2020;10:1664. https://doi.org/10.3389/ fphar.2019.01664.

21. Clarke JR, Ribeiro FC, Frozza RL, De Felice FG, Lourenco MV. Metabolic dysfunction in Alzheimer's disease: from basic neurobiology to clinical approaches. J Alzheimers Dis. 2018;64(s1):S405-S26. https://doi.org/10.3233/ JAD-179911.

22. Gagalo I, Rusiecka I, Kocic I. Tyrosine kinase inhibitor as a new therapy for ischemic stroke and other neurologic diseases: is there any hope for a better outcome? Curr Neuropharmacol. 2015;13(6):836-44.

23. Kehoe PG. The coming of age of the angiotensin hypothesis in Alzheimer's disease: progress toward disease prevention and treatment? J Alzheimers Dis. 2018;62(3):1443-66. https://doi.org/10.3233/JAD-171119.

24. Candasamy M, Elhassan SA, Bhattamisra SK, Wong YH, Lim MS, Busthamin NA, Mohamad IIni NB, Ng SS, Lim B, Ng SY, Ng WY. Type 3 diabetes (Alzheimer's disease): new insight for promising therapeutic avenues. Panminerva Med. 2020. https://doi.org/10.23736/S0031-0808.20.03879-3.

25. Ihara M, Saito S. Drug repositioning for Alzheimer's disease: finding hidden clues in old drugs. J Alzheimers Dis. 2020;74(4):1013-28. https://doi.org/10. 3233/JAD-200049.

26. Cummings J, Mintzer J, Brodaty H, Sano M, Banerjee S, Devanand DP, et al. Agitation in cognitive disorders: International Psychogeriatric Association provisional consensus clinical and research definition. Int Psychogeriatr. 2015;27(1):7-17. https://doi.org/10.1017/S1041610214001963.

27. Thomas T, Miners S, Love S. Post-mortem assessment of hypoperfusion of cerebral cortex in Alzheimer's disease and vascular dementia. Brain. 2015; 138:1059-69. https://doi.org/10.1093/brain/awv025.

28. Sakurai H, Hanyu H, Sato T, Kume K, Hirao K, Kanetaka H, et al. Effects of cilostazol on cognition and regional cerebral blood flow in patients with Alzheimer's disease and cerebrovascular disease: a pilot study. Geriatr Gerontol Int. 2013;13(1):90-7. https://doi.org/10.1111/j.1447-0594.2012.00866.x.

29. Lee JH, Park SY, Shin HK, Kim CD, Lee WS, Hong KW. Protective effects of cilostazol against transient focal cerebral ischemia and chronic cerebral hypoperfusion injury. CNS Neurosci Ther. 2008;14(2):143-52. https://doi.org/ 10.1111/j.1527-3458.2008.00042x.

30. Ono K, Tsuji M. Pharmacological potential of cilostazol for Alzheimer's disease. Front Pharmacol. 2019:10:559. https://doi.org/10.3389/fphar.2019.00559.

31. Calsolaro V, Edison P. Neuroinflammation in Alzheimer's disease: current evidence and future directions. Alzheimers Dement. 2016;12(6):719-32. https://doi.org/10.1016/j.jalz.2016.02.010.

32. Cerovic M, Forloni G, Balducci C. Neuroinflammation and the gut microbiota: possible alternative therapeutic targets to counteract Alzheimer's disease? Front Aging Neurosci. 2019;11:284. https://doi.org/10. 3389/fnagi.2019.00284

33. Mustapic M, Tran J, Craft S, Kapogiannis D. Extracellular vesicle biomarkers track cognitive changes following intranasal insulin in Alzheimer's disease. J Alzheimers Dis. 2019;69(2):489-98. https://doi.org/10.3233/JAD-180578.

34. Shoaib M, Kamal MA, Rizvi SMD. Repurposed drugs as potential therapeutic candidates for the management of Alzheimer's disease. Curr Drug Metab. 2017;18(9):842-52. https://doi.org/10.2174/1389200218666170607101622.

35. Kumar S, Chowdhury S, Kumar S. In silico repurposing of antipsychotic drugs for Alzheimer's disease. BMC Neurosci. 2017;18(1):76. https://doi.org/ 10.1186/s12868-017-0394-8.

36. Paranjpe MD, Taubes A, Sirota M. Insights into computational drug repurposing for neurodegenerative disease. Trends Pharmacol Sci. 2019; 40(8):565-76. https://doi.org/10.1016/j.tips.2019.06.003.

37. Zhang XZ, Quan Y, Tang GY. Medical genetics-based drug repurposing for Alzheimer's disease. Brain Res Bull. 2015;110:26-9. https://doi.org/10.1016/j. brainresbull.2014.11.003.

38. Zhang M, Schmitt-Ulms G, Sato C, Xi Z, Zhang Y, Zhou Y, et al. Drug repositioning for Alzheimer's disease based on systematic 'omics' data mining. PLoS One. 2016;11(12):e0168812. https://doi.org/10.1371/journal. pone.0168812

39. Fang J, Pieper AA, Nussinov R, Lee G, Bekris L, Leverenz JB, et al. Harnessing endophenotypes and network medicine for Alzheimer's drug repurposing. Med Res Rev. 2020. https://doi.org/10.1002/med.21709 Online ahead of print.

40. Paik H, Chung AY, Park HC, Park RW, Suk K, Kim J, et al. Repurpose terbutaline sulfate for amyotrhopic lateral sclerosis using electronic medical records. Sci Rep. 2015;5:8580. https://doi.org/10.1038/srep08580. 
41. Koenig AM, Mechanic-Hamilton D, Xie SX, Combs MF, Cappola AR, Xie L, et al. Effects of the insulin sensitizer metformin in Alzheimer disease: pilot data from a randomized placebo-controlled crossover study. Alzheimer Dis Assoc Disord. 2017:31(2):107-13. https://doi.org/10.1097/WAD. 0000000000000202.

42. Kile S, Au W, Parise C, Rose K, Donnel T, Hankins A, et al. IVIG treatment of mild cognitive impairment due to Alzheimer's disease: a randomised double-blinded exploratory study of the effect on brain atrophy, cognition and conversion to dementia. J Neurol Neurosurg Psychiatry. 2017;88(2):10612. https://doi.org/10.1136/jnnp-2015-311486.

43. Stonnington CM, Harel B, Locke DEC, Hentz JG, Zhang N, Maruff P, et al. Lorazepam challenge for individuals at varying genetic risk for Alzheimer disease. Alzheimer Dis Assoc Disord. 2017;31(4):271-7. https://doi.org/10. 1097/WAD.0000000000000200.

44. Lawlor B, Segurado R, Kennelly S, Olde Rikkert MGM, Howard R, Pasquier F, et al. Nilvadipine in mild to moderate Alzheimer disease: a randomised controlled trial. PLoS Med. 2018;15(9):e1002660. https://doi.org/10.1371/ journal.pmed.1002660

45. Boada M, Lopez O, Nunez L, Szczepiorkowski ZM, Torres M, Grifols C, et al. Plasma exchange for Alzheimer's disease Management by Albumin Replacement (AMBAR) trial: study design and progress. Alzheimers Dement (N Y). 2019;5:61-9. https://doi.org/10.1016/.jtrci.2019.01.001.

46. Fleminger J, Goldacre B. Prevalence of clinical trial status discrepancies: a cross-sectional study of 10,492 trials registered on both ClinicalTrials.gov and the European Union Clinical Trials Register. PLoS One. 2018;13(3): e0193088. https://doi.org/10.1371/journal.pone.0193088 eCollection 2018.

47. Clyne B, Boland F, Murphy N, Murphy E, Moriarty F, Barry A, et al. Quality, scope and reporting standards of randomised controlled trials in Irish Health Research: an observational study. Trials. 2020;21(1):494. https://doi. org/10.1186/s13063-020-04396-X.

48. Strzebonska K, Wasylewski MT, Zaborowska L, Riedel N, Wieschowski S, Stretch $D$, et al. Results dissemination of registered clinical trials across polish academic institutions: a cross-sectional analysis. BMJ Open. 2020; 10(1):e034666. https://doi.org/10.1136/bmjopen-2019-034666.

49. Pillamarapu M, Mohan A, Saberwal G. An analysis of deficiencies in the data of interventional drug trials registered with clinical trials registry - India. Trials. 2019;20(1):535. https://doi.org/10.1186/s13063-019-3592-0.

50. Pandolfini C, Bonati M. An audit to evaluate an Institute's Lead Researchers' knowledge of trial registries and to investigate adherence to data transparency issues in an Italian research institute registry. Trials. 2018;19(1): 509. https://doi.org/10.1186/s13063-018-2910-2.

51. Cummings J, Isaacson S, Mills R, Williams H, Chi-Burrs K, Corbett A, et al. Pimavanserin for patients with Parkinson's disease psychosis: a randomised, placebo-controlled phase 3 trial. Lancet. 2014;383(9916):533-40. https://doi. org/10.1016/S0140-6736(13)62106-6

52. Cummings J, Ballard C, Tariot P, Owen R, Foff E, Youakim J, et al. Pimavanserin: potential treatment for dementia-related psychosis. J Prev Alzheimers Dis. 2018;5(4):253-8. https://doi.org/10.14283/jpad.2018.29.

53. Grossberg GT, Kohegyi E, Mergel V, Josiassen MK, Meulien D, Hobart M, et al. Efficacy and safety of brexpiprazole for the treatment of agitation in Alzheimer's dementia: two 12-week, randomized, double-blind, placebocontrolled trials. Am J Geriatr Psychiatry. 2019. https://doi.org/10.1016/j.jagp. 2019.09.009.

54. Tartaglia LA. Complementary new approaches enable repositioning of failed drug candidates. Expert Opin Investig Drugs. 2006;15(11):1295-8. https://doi. org/10.1517/13543784.15.11.1295

55. Baek MC, Jung B, Kang H, Lee HS, Bae JS. Novel insight into drug repositioning: Methylthiouracil as a case in point. Pharmacol Res. 2015;99: 185-93. https://doi.org/10.1016/j.phrs.2015.06.008.

\section{Publisher's Note}

Springer Nature remains neutral with regard to jurisdictional claims in published maps and institutional affiliations.

Ready to submit your research? Choose BMC and benefit from:

- fast, convenient online submission

- thorough peer review by experienced researchers in your field

- rapid publication on acceptance

- support for research data, including large and complex data types

- gold Open Access which fosters wider collaboration and increased citations

- maximum visibility for your research: over $100 \mathrm{M}$ website views per year

At BMC, research is always in progress.

Learn more biomedcentral.com/submissions 